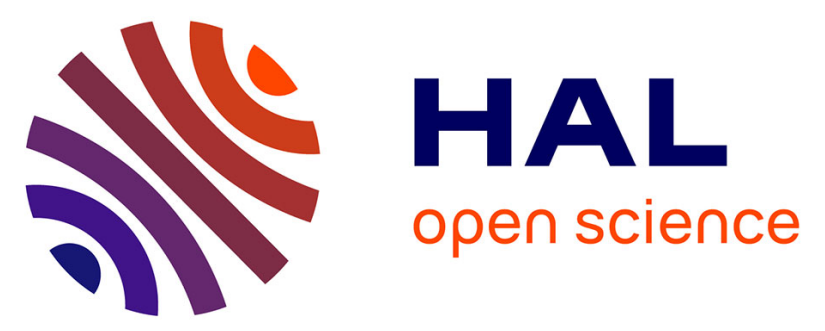

\title{
LES TARIFICATIONS AU COÛT MARGINAL VERSUS COÛT MOYEN FACE À DES CHOCS CLIMATIQUES AU SÉNÉGAL: UN MODĖLE DYNAMIQUE D'ÉQUILIBRE GÉNÉRAL CALCULABLE APPLIQUÉ À L'EAU
}

\author{
Anne Briand
}

\section{To cite this version:}

Anne Briand. LES TARIFICATIONS AU COÛT MARGINAL VERSUS COÛT MOYEN FACE À DES CHOCS CLIMATIQUES AU SÉNÉGAL: UN MODÈLE DYNAMIQUE D'ÉQUILIBRE GÉNÉRAL CALCULABLE APPLIQUÉ À L'EAU. Economie et Prévision, 2008. hal-02430398

\author{
HAL Id: hal-02430398 \\ https://hal.science/hal-02430398
}

Submitted on 7 Jan 2020

HAL is a multi-disciplinary open access archive for the deposit and dissemination of scientific research documents, whether they are published or not. The documents may come from teaching and research institutions in France or abroad, or from public or private research centers.
L'archive ouverte pluridisciplinaire HAL, est destinée au dépôt et à la diffusion de documents scientifiques de niveau recherche, publiés ou non, émanant des établissements d'enseignement et de recherche français ou étrangers, des laboratoires publics ou privés. 


\section{LES TARIFICATIONS AU COÛT MARGINAL VERSUS COÛT MOYEN FACE À DES CHOCS CLIMATIQUES AU SÉNÉGAL : UN MODĖLE DYNAMIQUE D'ÉQUILIBRE GÉNÉRAL CALCULABLE APPLIQUÉ À L'EAU »}

\section{Anne Briand}

La Documentation française | «Économie \& prévision »

2008/4 n 185 | pages 103 à 122

ISSN 0249-4744

Article disponible en ligne à l'adresse :

https://www.cairn.info/revue-economie-et-prevision-2008-4-page-103.htm

Distribution électronique Cairn.info pour La Documentation française.

(C) La Documentation française. Tous droits réservés pour tous pays.

La reproduction ou représentation de cet article, notamment par photocopie, n'est autorisée que dans les limites des conditions générales d'utilisation du site ou, le cas échéant, des conditions générales de la licence souscrite par votre établissement. Toute autre reproduction ou représentation, en tout ou partie, sous quelque forme et de quelque manière que ce soit, est interdite sauf accord préalable et écrit de l'éditeur, en dehors des cas prévus par la législation en vigueur en France. Il est précisé que son stockage dans une base de données est également interdit. 


\title{
Les tarifications au coût marginal versus coût moyen face à des chocs climatiques au Sénégal : un modèle dynamique d'équilibre général calculable appliqué à l'eau »
}

Anne Briand $^{(*)}$

\begin{abstract}
Avec une forte croissance de la demande en eau, les gouvernements sénégalais ont d'abord tenté de faire face à la rareté en s'engageant, à la fin des années quatre vingt dix, dans de nombreux programmes d'investissements pour accroître les capacités de production. Parallèlement, l'idée d'une rationalisation de l'utilisation de la ressource par une meilleure gestion de la demande s'est développée. C'est pourquoi, une politique tarifaire de l'eau a rapidement accompagné la politique de l'offre. Il s'en est suivi une augmentation du prix de l'eau potable de 3\% par an (en monnaie courante) entre 1996 et 2003. Cette hausse tarifaire visait aussi l'atteinte de l'équilibre budgétaire du secteur. Cependant, elle interroge sur ses conséquences sur les différents secteurs notamment agricoles qui emploient une partie importante de la population active ainsi que sur l'accès à l'eau potable des ménages les plus pauvres.
\end{abstract}

Par ailleurs, malgré une politique volontariste de lutte contre les désajustements offre/demande, le Sénégal devrait connaître à partir de 2015 des déficits hydriques importants, compte tenu de l'évolution de la demande (croissance économique) et de la croissance démographique. Ces déficits hydriques pourraient être pires encore si des aléas climatiques conduisaient à une baisse de la pluviométrie (sécheresse).

Pour capter l'ensemble des effets microéconomiques et macroéconomiques de la disponibilité de la ressource, d'une part, et de la politique tarifaire de l'eau, d'autre part, nous proposons un modèle dynamique séquentiel d'équilibre général calculable (EGC) prenant en compte les évolutions de long terme de la disponibilité de la ressource primaire (l'offre) au regard de la croissance démographique (la demande). Le modèle montre comment l'économie soumise à des risques futurs de pénuries d'eau, amortit le choc à long terme selon qu'elle maintient sa politique actuelle de tarification au coût moyen (équilibre budgétaire du secteur) ou qu'elle instaure une tarification au coût marginal (via l'octroi d'une subvention de l'État pour la survie $d u$ secteur). Les originalités du modèle reposent sur la modélisation d'un secteur de production d'eau potable en monopole naturel ainsi que sur une désagrégation fine du marché de distribution (branchement privé, borne fontaine et informel) et des usages de l'eau.

Plus précisément, le modèle simule à court terme une phase d'accroissement de la disponibilité de la ressource (justifiée par les politiques d'accroissement de l'offre et au contexte

(*) Maître de conférences, Care (Centre d'analyse et de recherche en économie), UFR Droit- Eco- Gestion, Université de Rouen. E-mail: anne.briand@univ-rouen.fr

Je remercie le professeur Olivier Beaumais pour ses remarques constructives ainsi que le professeur Bernard Decaluwé pour ses commentaires très précieux lors de mon séjour de recherche au Cirpée (Université Laval, Québec). 
pluviométrique actuel plutôt favorable) suivie d'une phase d'apparition de déficits hydriques conformément aux prévisions d'évolution de la demande future et des risques de nouvelles sécheresses au Sénégal. Ces chocs climatiques sont simulées de façon combinée à deux politiques tarifaires de gestion de la demande en eau : tarification au coût moyen versus tarification au coût marginal.

Les résultats montrent que si, à court terme, une tarification au coût marginal est plus bénéfique qu'une tarification au coût moyen, elle ne fait qu'amplifier positivement les effets sans les inverser. En effet dans les deux cas, l'accroissement de court terme de la disponibilité de la ressource primaire génère une expansion de l'économie avec une baisse du taux de chômage, une hausse de l'investissement et du bien-être total. Dans les deux cas, la distribution d'eau potable à la borne fontaine se développe plus que celle au branchement privé et le formel se substitue à l'informel. L'expansion des secteurs agricoles est plus forte lorsque la politique d'accroissement de l'offre s'accompagne de la mise en place d'une tarification au coût marginal. Cette dernière apparaît plus favorable à la lutte contre l'insécurité alimentaire. Enfin le bien-être des différentes catégories de ménages s'accroît davantage aussi dans le deuxième scénario. En revanche, les résultats de long terme sont plus intéressants puisqu'ils montrent que les effets d'une tarification au coût marginal sont bien plus bénéfiques. En effet, lorsque le Sénégal fait face à des déficits hydriques, elle permet de mieux gérer la rareté de l'eau en inversant complètement les tendances de long terme de l'économie qui maintiendrait une tarification au coût moyen. Plus précisément, l'investissement total s'accrô̂t alors qu'il diminuait dans le premier scénario. Suite au choc de rareté de la ressource, le bien-être total se dégrade moins que dans le premier cas. Plus particulièrement, la mise en place d'une tarification au coût marginal permet d'atténuer les effets négatifs des pénuries d'eau sur le bien-être des ménages. L'expansion de la plupart des secteurs permise par le passage à une tarification au coût marginal relance l'activité économique en diminuant le taux de chômage tandis que ce dernier augmentait avec la tarification au coût moyen. Malgréla pénurie d'eau, le passage à une tarification au coût marginal permet d'accrô̂tre la production agricole du riz. pluvial et du maraîchage alors que tous les secteurs agricoles reculent lorsqu'on maintient une tarification au coût moyen. Cette réforme tarifaire serait plus favorable à la lutte contre l'insécurité alimentaire. Enfin en terme d'approvisionnement en eau potable, on observe une tendance inverse au premier scénario puisque le déficit hydrique accompagné d'une politique tarifaire au coût marginal permet malgré tout une expansion de la distribution d'eau potable (branchement privé et borne fontaine) grâce à la dynamique d'investissement, ce qui accrô̂t l'accès des ménages au service. Enfin le secteur informel ne joue plus le rôle de régulateur de la pénurie puisqu'il recule.

En conclusion, la tarification au coût marginal permet une meilleure gestion à long terme de la rareté de la ressource et de lutter contre l'insécurité alimentaire même si l'État doit intervenir en subventionnant le secteur de production d'eau potable (monopole naturel). Elle contrecarre les effets négatifs des déficits hydriques puisque la baisse du coût de l'intrant eau combinée à la hausse de l'investissement total (grâce à la hausse de l'épargne des agents domestiques et étrangers plus forte que la dégradation de l'épargne gouvernementale), relance l'activité économique et améliore le bien être total.

Ce résultat va à l'encontre des directives des bailleurs de fond qui imposent aux sociétés d'eau des pays en développement la contrainte d'équilibre budgétaire via une tarification au coût moyen. Le modèle montre que l'intervention de l'État via une subvention visant à combler le déficit du secteur lié à une tarification au coût marginal ne génère pas, dans le cadre d'une économie appliquée avec fiscalité distordante, d'effets négatifs. Cependant, ce résultat reste lié aux hypothèses de bouclage du modèle et en particulier à l'endogénéité de la balance courante qui rend en partie possible le financement de la politique intérieure par l'étranger. Il serait intéressant de fixer la balance courante dans le modèle et d'endogénéiser une taxe qui viserait le financement de la subvention. 
La vulnérabilité climatique des économies d'Afrique subsaharienne est principalement liée au problème de disponibilité des ressources en eau. Comme pour le reste du Sahel, 1968 marque le début d'une longue période de sécheresse au Sénégal (1968-1998). Celle-ci a fortement perturbé les activités économiques surtout dans le monde rural. Elle a entraîné plusieurs conséquences très dommageables : une baisse de la production agricole, un dérèglement du calendrier cultural, la famine, l'exode vers les villes, une forte mortalité du cheptel, un assèchement des cours d'eau et, l'avancée du désert. Depuis 1999, le Sénégal connaît un retour des années humides et des productions agricoles plus importantes. Mais le retour des pluies sur plusieurs années consécutives n'autorise pas a priori à considérer qu'il s'agit de la fin de la période de sécheresse. De plus malgré un retour des pluies, la longue période de sécheresse a entraîné le renforcement de la variabilité climatique par une accentuation des phénomènes extrêmes. Enfin, des projections montrent que le Sénégal devrait encore faire face dans le futur à un risque de désajustement entre l'offre et la demande notamment, en terme d'alimentation en eau potable (Management System Consultants Corp, 1998 ; Ndaw, 2005 ; SDE, 2004).

Face à la contrainte d'une forte variabilité de la pluviométrie qui accroît le risque de déficit hydrique, le Sénégal a choisi de réformer en 1995 le secteur de l'eau afin de mettre en place une politique volontariste de lutte contre les désajustements offre/demande. Avec des prévisions d'une forte croissance de la demande en eau, les gouvernements sénégalais se sont alors engagés dans des programmes d'investissements pour accroître les capacités de production. Parallèlement, l'idée d'une rationalisation de l'utilisation de la ressource par une meilleure gestion de la demande s'est développée. C'est pourquoi, une politique tarifaire de l'eau potable a rapidement accompagné la politique d'offre. Il s'en est suivi une augmentation du prix de l'eau potable de 3\% par an entre 1996 et 2003 (en monnaie courante). Cette hausse tarifaire visait à la fois l'atteinte de l'équilibre budgétaire du secteur mais aussi la volonté de rationaliser la demande. Cependant, elle interroge sur ses conséquences directes et indirectes sur l'ensemble de l'économie sénégalaise.

Pour capter tous les effets de rétroaction de la disponibilité de la ressource et de la politique de tarification de l'eau sur le long terme, nous proposons un modèle dynamique séquentiel d'équilibre général calculable (EGC) prenant en compte les évolutions de long terme de la disponibilité de la ressource primaire (l'offre) au regard de la croissance démographique (la demande). Le modèle montre comment l'économie soumise à des risques futurs de pénuries d'eau, amortit le choc à long terme selon qu'elle maintient sa politique actuelle de tarification au coût moyen (équilibre budgétaire du secteur) ou qu'elle instaure une tarification au coût marginal (via l'octroi d'une subvention de l'Etat pour la survie du secteur). Plus précisément, il décrit les mécanismes par lesquels l'évolution des différents prix de l'eau (branchement privé, borne-fontaine et informel) affecte d'une part, les secteurs utilisateurs de la ressource (agricoles, industriels et services) et d'autre part, les ménages (Dakar, autres centres urbains et ruraux). Il met en évidence les effets de substitution entre les différents modes d'approvisionnement en eau potable. Enfin, le modèle évalue les impacts à court et à plus long terme sur la sécurité alimentaire (production agricole), le chômage, l'investissement et le bien être total de chacune de ces tarifications.

La première section de l'article présente les politiques menées au Sénégal pour lutter contre les risques de désajustements offre/demande. La seconde section présente, au regard de la littérature, les originalités du modèle EGC et des simulations développées. La troisième section présente la matrice de comptabilité sociale et la quatrième le modèle. Enfin, la dernière section présente les résultats des chocs climatiques combinés aux deux politiques de gestion de la demande et de la rareté (tarification au coût moyen versus tarification au coût marginal).

\section{Les politiques de lutte contre les risques de désajustements offre/demande d'eau}

\section{Réforme du secteur dans un contexte de pénurie}

La réforme de secteur de l'eau au Sénégal (1995) a été introduite dans un contexte de pénurie d'eau à Dakar qui a duré des années sans qu'une solution rapide ne pût être trouvée. Cette pénurie d'eau était non seulement liée à l'insuffisance des infrastructures de production et de distribution d'eau potable, mais aussi à un déficit pluviométrique. Or, cette pénurie d'eau à Dakar était très dommageable pour l'ensemble de l'économie sénégalaise (poids de la capitale dans l'activité économique du Sénégal). Les efforts réalisés dans les années quatre-vingt en matière d'alimentation en eau potable dans la capitale se sont révélés très rapidement insuffisants du fait que les nappes d'eau proches de Dakar étaient surexploitées. Compte tenu de la hausse de la demande, il a fallu chercher de l'eau de plus en plus loin mais moyennant des coûts de transport et de distribution de plus en plus importants. Les besoins d'investissement nécessaires, uniquement pour augmenter la production et faire face à la demande urgente de l'époque, étaient évalués à plus de 60 milliards de FCFA ${ }^{(1)}$ de l'époque. Parallèlement, la société d'eau de l'époque (Sonees ${ }^{(2)}$ ) rencontrait de graves problèmes de trésorerie qui l'empêchait d'assurer correctement le service d'eau : ses recettes 
issues de la vente d'eau étaient trop faibles et insuffisamment collectées (faible taux de recouvrement des factures). Aussi, le Sénégal s'est engagé dans une réforme visant à intégrer le secteur privé dans la production et la distribution d'eau potable. Ce partenariat public-privé (PPP) associe alors l'État sénégalais, la Sones ${ }^{(3)}$ (société publique de patrimoine) et la $\mathrm{Sde}^{(4)}$ (société privée). Les objectifs du partenariat sont alors clairement définis : résorber le déficit en eau potable via les investissements, accroître l'accès des ménages et atteindre l'équilibre budgétaire du secteur à l'horizon 2003.

\section{Le programme de renforcement des ressources en eau}

Pour ce faire, la réforme s'est immédiatement accompagnée de la mise en place d'une politique d'accroissement de l'offre comportant deux volets : le renforcement des ressources en eau via l'accroissement des capacités de production disponibles de Dakar et la distribution du service d'eau potable aux ménages. Dans le cadre de cet article, nous mettons l'accent sur le premier volet (voir Briand (2006b) pour le second). Le renforcement des ressources en eau s'est opéré grâce à deux grands programmes d'investissement : le Projet sectoriel eau (PSE) et le Plan sectoriel à long terme (PLT). Le PSE (1996-2003) visait simplement à rattraper le déficit de production d'eau potable de l'époque. Les investissements engagés (216 milliards de $\mathrm{FCFA}^{(5)}$ ) ont permis d'augmenter les capacités de production de 34\% (Sde, 2004). Mais il s'est avéré à l'issue de trois ans de programme que les investissements ne permettaient que de couvrir le déficit en eau de l'époque et que ce dernier réapparaîtrait dès 2003. C'est pourquoi un second programme a été engagé, le PLT (2003-2007), afin d'anticiper sur la demande future en évitant que de nouveaux désajustements offre/demande ne réapparaissent. Ce dernier prévoit alors l'engagement de 300 milliards de FCFA $^{(6)}$ pour accroître à nouveau les capacités de production ${ }^{(7)}$ (Sde, 2004).

\section{Les prévisions de pénuries futures d'eau}

En dépit des investissements engagés pour l'extension des capacités de production, des déficits hydriques menaceraient le Sénégal à l'horizon 2015. Une étude ${ }^{(8)}$ (Management System Consultants Corp, 1998) a établi une projection de la demande en eau potable du Sénégal sur une période de 30 ans. Ces projections définissent les besoins en eau futurs compte tenu de la croissance démographique et de la croissance économique qui induirait une utilisation plus intensive de l'eau. Dans l'hypothèse moyenne, l'étude démontre qu'à l'horizon 2030, la capitale devrait connaître un doublement de la population, une hausse de $50 \%$ de la consommation domestique d'eau potable (aux branchements privés, aux bornes fontaines et autres) et, un triplement de la consommation d'eau industrielle et commerciale. $\mathrm{Au}$ total, la consommation d'eau à Dakar (hors irrigation) devrait plus que doubler. Des évolutions semblables devraient marquer les autres régions du Sénégal. D'où au total, un doublement de la demande en eau potable du Sénégal à l'horizon 2030. L'étude démontre alors qu'en adoptant un taux de disponibilité des équipements de production ${ }^{(9)}$ de $100 \%$, un déficit apparaîtrait en 2015 et ce, malgré la mise en place du PLT. Le graphique 1 montre qu'en 2015 la production nécessaire pour répondre à la demande serait supérieure aux capacités d'offre : d'où l'apparition d'un désajustement offre/demande.

\section{Graphique 1 : les désajustements offre/demande d'eau futurs}
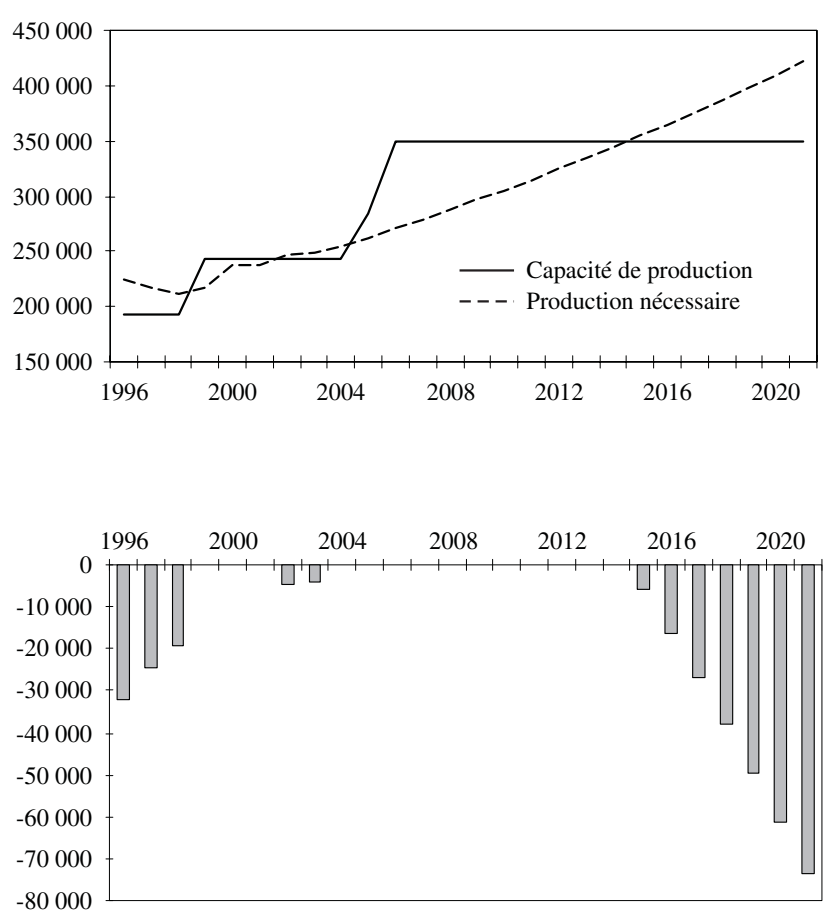

Source : Sde (2004).

Ce déficit hydrique pourrait être encore plus important si la pluviométrie venait à se dégrader. En effet, compte tenu de la forte variabilité pluviométrique qui affecte le Sénégal, le risque d'une sécheresse future à l'horizon 2015 n'est pas écarté. Et ceci, en dépit d'une amélioration relative des pluies ces dernières années. Compte tenu des risques d'apparition de pénuries d'eau et de l'évolution de la demande croissante, la réforme du secteur s'est aussi s'accompagnée de la mise en place d'une politique de gestion de la demande.

\section{La politique de gestion de la demande}

Les prévisions de désajustements offre/demande ont motivé la mise en place d'une politique de gestion de la demande. Le premier objectif défini par la Sones a été l'atteinte en 2003 de l'équilibre budgétaire du 
Tableau 1 : évolution du prix moyen de l'eau potable $\left(\mathrm{FCFA} / \mathrm{m}^{3}\right)$

\begin{tabular}{|l|c|c|c|c|c|c|c|c|}
\hline \multicolumn{1}{|c|}{ FCFA $/ \mathrm{m}^{3}$} & 1996 (mai) & 1997 & 1998 & 1999 & 2000 & 2001 & 2002 & Evolution 96-02 \\
\hline Prix moyen TTC & 380 & 396 & 414 & 430 & 450 & 453 & 456 & $3,10 \%$ \\
dont taxes (TVA et FNH) & 29 & 30 & 38 & 37 & 41 & 41 & 41 & $6,10 \%$ \\
\hline
\end{tabular}

Source : Sde (2004)

secteur hors Projet long terme via une augmentation annuelle du prix de l'eau (en monnaie courante). Le tableau 1 montre que celle-ci a été de 3,1\% par an entre 1996 et 2003. Ainsi, l'objectif d'équilibre budgétaire a été atteint.

Le second objectif poursuivi par la Sones est la lutte contre la pauvreté. C'est pourquoi, le Sénégal comme la plupart des pays en développement, a adopté une tarification progressive de l'eau potable (Brocklehurst, Janssens, 2004). L'idée sous-jacente est d'assurer pour tous l'accès à une quantité minimale d'eau à un coût raisonnable. Cette structure tarifaire consiste à facturer aux ménages les premiers $\mathrm{m} 3$ à un prix très faible, afin de répondre aux besoins vitaux (boisson, hygiène). Cette première tranche qualifiée de «sociale » est conçue de façon à subventionner la consommation des ménages les plus pauvres par celle des plus riches. Puis les tranches suivantes de consommation (de confort), sont facturées à un prix plus élevé. Enfin les consommations au dessus d'un certain seuil sont tarifées à un prix dissuasif, afin de décourager le gaspillage d'une ressource limitée (voir Briand (2006b) pour plus de détails).

Les résultats d'une enquête que nous avons réalisée à Dakar en 2005 (Briand, 2006b) nous amènent à nous demander si la question préalable qui doit être posée ne serait pas celle du choix du tarif de base (coût moyen) sur lequel la structure progressive du prix de l'eau repose. En effet, le prix de l'eau au branchement privé apparaît trop élevé pour de nombreux ménages (Briand, 2006b). Le prix moyen cible qui a été défini en 1996 puis progressivement atteint en 2003 apparaît élevé au regard des autres pays d'Afrique. Il est proche de celui rencontré dans les pays européens. Un tel tarif moyen est d'ailleurs rarement rencontré en Afrique de l'Ouest (Sones, 2002). Le passage à un tarif de base au coût marginal, qui signifierait une baisse du prix, ne permettrait-il pas d'accroître l'accès à l'eau potable et le bien être des ménages?

C'est pourquoi, bien que la question du choix de la structure tarifaire progressive soit importante en terme de redistribution des revenus, nous nous concentrons, dans cet article, sur la question du choix du tarif de base sur lequel cette structure repose (coût moyen versus coût marginal).

\section{Les originalités du modèle EGC au regard de la littérature}

\section{La littérature}

Dans les modèles EGC appliqués au Sénégal ${ }^{(10)}$, seuls les travaux de Cabral (2005) intègrent les ressources en eau. L'auteur les considère comme un facteur de production utilisé par l'agriculture à la lumière d'autres travaux (Berck et alii, 1991 ; Robinson et Gehlar, 1995 ; Goodman, 2000 ; Gomez et alii, 2004 et Thabet, 2003). Nous retenons cette hypothèse. Cependant dans notre modèle, la ressource primaire est un facteur de production utilisé d'une part par l'agriculture mais aussi, par le secteur de production d'eau potable dont le rôle est de transformer cette eau primaire en eau potable.

La question de l'eau n'a été le plus souvent étudiée que dans le cadre de modèles EGC appliqués à l'agriculture. La littérature montre qu'il existe principalement deux façons d'aborder la problématique de l'eau dans ces modèles.

Tout d'abord et majoritairement, des modèles EGC ont été construits afin de simuler des politiques d'offre d'eau potable. Ces modèles abordent la question de l'eau selon une approche par les quantités. Certains mettent en évidence les impacts de politiques visant à accroître la disponibilité de la ressource ou analysent les conséquences d'une baisse du stock d'eau (Berck et alii, 1991 ; Robinson et Gehlar, 1995 ; Löfgren et Robinson, 1997). La limite de ces modèles est que l'eau n'est consommée que par l'agriculture et non par les autres secteurs et les ménages. C'est pourquoi d'autres modèles se sont concentrés sur la question de la réallocation de l' eau entre les différents usages (Seung et alii, 2000; Goodman, 2000 ; Gomez et alii, 2004). Il est intéressant de noter que les modèles développés par Berck et alii, (1991), Robinson et Gehlar (1995), Löfgren et Robinson (1997) et Gomez et alii, (2004) reposent tous sur l'hypothèse d'une offre d'eau exogène. Dans celui de Gomez et alii, (2004), appliqué aux Iles Baléares, le stock d'eau (qui dépend du niveau de l'offre de la ressource primaire) est déterminé par le degré de sécheresse. Nous retenons dans notre modèle cette hypothèse d'un stock d'offre exogène de la ressource primaire. Enfin, Goodman (2000) introduit dans la fonction de production agricole une substitution entre la terre et l'eau, hypothèse que nous retenons dans notre modèle. 
La seconde catégorie de modèles EGC aborde explicitement la question des outils tarifaires comme politique de gestion de la demande. Ces modèles, beaucoup plus rares, abordent la question de l'eau selon une approche par les prix. En effet, c'est dans un contexte de rareté croissante de la ressource que ces derniers cherchent à simuler des politiques tarifaires de gestion de la demande en eau potable (Horridge et alii, (1993) ; Decaluwé et alii, (1998, 1999, 2001); Thabet (2003)). Dans les deux derniers travaux, le premier apport est d'avoir introduit une demande d'eau potable des secteurs agricoles, industriels, des services (en tant que bien intermédiaire) et des ménages (en tant que bien final), générant alors de véritables conflits d'usages de la ressource, notamment suite aux chocs de tarification qu'ils opèrent. Leur second apport est d'avoir explicitement modélisé un secteur de production d'eau potable, ce qui est très rare dans la littérature. Thabet (2003) modélise un secteur de production et de distribution d'eau potable en adoptant les hypothèses suivantes : tout d'abord, il considère que ce secteur achète auprès de l'État de l'eau primaire (hypothèse que nous retenons dans notre modèle). Cette eau est par la suite transformée en eau potable selon un processus de production conventionnel : l'eau primaire est combinée en proportions fixes avec l'agrégat issu d'une fonction CES entre le capital et le travail salarié pour former la valeur ajoutée du secteur. Cette dernière est utilisée en proportions fixes avec les autres intrants intermédiaires pour produire l'eau potable. Decaluwé et alii, $(1998 ; 1999 ; 2001)$ ont, à travers la fonction de Weibull, élaboré une technologie de production d'eau originale rendant compte de l'exploitation des eaux souterraines. L'eau potable est produite à partir de deux technologies : la première met à disposition des usagers l'extrant eau à partir des barrages tandis que la deuxième repose sur l'eau issue du pompage et du creusement des puits. Cependant, aucun des deux modèles ne tient compte de la particularité de la structure du marché de l'eau (monopole naturel à rendements d'échelle croissants). De plus, ces études n'ont porté que sur la question de la tarification de l'eau à usage agricole. Par ailleurs, les modèles développés par ces auteurs ne sont que des modèles statiques.

Au regard de cette littérature, pour traiter de la question de la gestion de la rareté croissante de l'eau au Sénégal, nous proposons un modèle EGC qui présente trois originalités :

- une représentation des spécificités du marché de distribution d'eau potable telles que révélées par notre enquête (Briand, 2006b) ;

- une approche dynamique séquentielle récursive tenant compte de l'évolution future de la demande (croissance démographique) et de la disponibilité des ressources en eau ;

- une modélisation d'un secteur de production d'eau potable en situation de monopole naturel (rendements d'échelle croissants) afin de comparer à court et à long terme, les effets des tarifications au coût moyen et au coût marginal de l'eau potable.

\section{Les originalités du modèle EGC}

\section{Une désagrégation de la distribution d'eau potable}

Compte tenu d'une part, des résultats de notre enquête (Briand, 2006b) qui montrent la coexistence importante de trois modes d'approvisionnement (branchement privé, borne fontaine et informel) et d'autre part, d'une forte hétérogénéité des prix de l'eau selon le mode, il apparaît essentiel de représenter dans le cadre EGC un marché de distribution d'eau potable avec ces trois segments.

C'est pourquoi le modèle destiné à mesurer les impacts de la variabilité climatique sur les ressources en eau et des politiques alternatives de gestion de la demande (tarification coût moyen versus coût marginal), intègre ces trois segments de distribution d'eau potable afin d'observer les effets de substitution entre les trois modes d'approvisionnement (branchement privé, borne fontaine et informel) suite aux chocs.

\section{Un scenario climatique élaboré grâce à une approche dynamique séquentielle récursive}

L'intérêt du modèle dynamique est de simuler un scenario climatique plus sophistiqué qu'en statique. Il permet de prendre en compte une dynamique d'évolution de la disponibilité de la ressource en eau rendant mieux compte de la réalité.

En effet, nous simulons sur un horizon de 20 ans :

- une première phase d'accroissement de la disponibilité de la ressource. Celle-ci se justifie à la fois par les politiques d'accroissement de l'offre qui permettent une plus grande mobilisation de la ressource primaire mais aussi, par la tendance récente à une hausse de la pluviométrie (les pluies de ces dernières années sont plus abondantes que la moyenne) ;

- une seconde phase caractérisée par une stabilité de la disponibilité de la ressource ;

- une troisième phase marquée par l'apparition de déficits hydriques. Celle-ci se justifie à la fois par les prévisions d'une demande future supérieure aux capacités d'offre (compte tenu de l'évolution démographique et du développement économique) mais aussi, par des risques futurs de sécheresse.

Un secteur de production d'eau potable en monopole naturel: tarification coût moyen versus coût marginal

La particularité de notre modèle est la modélisation d'un secteur de production d'eau potable en monopole naturel. En effet, ce secteur est caractérisé par l'existence d'économies d'échelle. Il s'agit d'une activité à rendements d'échelle croissants, cas particulier du monopole naturel. La théorie économique nous enseigne que dans le cas d'un monopole qui produit un seul type de bien, les 
rendements d'échelle croissants impliquent que le coût moyen à long terme est décroissant (économies d'échelle). Ainsi, le coût marginal est toujours inférieur au coût moyen. La tarification optimale qui égalise le prix et le coût marginal conduit alors inéluctablement à un déficit du monopole. Celui-ci doit être comblé par des subventions financées le plus souvent par l'impôt. Mais ces subventions visant à résorber le déficit d' un monopole public sont souvent mal perçues, même si ce déficit est justifié par le critère d'optimalité collective que représente la tarification au coût marginal. De plus, le prélèvement fiscal qui permet de les financer peut avoir des conséquences dommageables sur le plan de l'équité ou conduire à modifier le comportement des ménages dans un sens non souhaitable (par exemple lorsqu' on modifie les taux de taxes indirectes). C'est la raison pour laquelle on considère souvent plus raisonnable d'astreindre le monopole public au respect d'une contrainte d'équilibre budgétaire : financer les coûts de production par des recettes au moins équivalentes. On a vu précédemment que la politique tarifaire définie au Sénégal prend clairement acte de cet objectif d'équilibre budgétaire du secteur. C'est pourquoi, nous considérons dans notre modèle EGC que la tarification de l'eau à l'année de référence est une tarification au coût moyen (équilibre budgétaire du secteur de production d'eau potable).

Cependant, comme il est connu dans la littérature, dans une économie stylisée (sans fiscalité préexistante), la tarification au coût marginal est le principe économique simple qui permet d'assurer une allocation optimale de la ressource entre tous les utilisateurs. La tarification au coût marginal fait en effet disparaître la perte sociale générée par une tarification au coût moyen et maximise le bien être. Nous souhaitons simuler ce scenario de tarification de l'eau dans un cadre EGC. Pour assurer la survie du secteur, nous considérons alors que cette tarification s'accompagne du versement par l'État d'une subvention à la société de production d'eau potable afin de financer son déficit. Compte tenu de la fiscalité pré-existante dans notre modèle EGC, nous n'introduisons pas de nouvelle taxe pour financer cette subvention. La question cruciale que nous nous posons est celle des effets en équilibre général (en présence d'une fiscalité distordante) de ces deux types de tarification de l' eau (coût moyen versus coût marginal). La première permet l'équilibre budgétaire du secteur sans intervention de l'État. Mais la seconde pourrait permettre d'accrô̂tre l'accès des ménages et le bien être total.

Ainsi, dans une économie soumise à des risques futurs de déficits hydriques, le modèle montre comment les politiques tarifaires de gestion de la demande, affectent l'économie sénégalaise à court et à plus long terme. Plus précisément, il décrit les mécanismes par lesquels l'évolution des différents prix de l'eau (branchement privé, borne-fontaine et informel) affecte d'une part, les secteurs utilisateurs de la ressource (agricoles, industriels et services) et d'autre part, les ménages (Dakar, autres centres urbains et ruraux). Il met en évidence la nouvelle répartition de la ressource entre les différents usages et plus particulièrement, les effets de substitution entre les différents modes d'approvisionnement en eau potable à usage intermédiaire ou final, par branche et par ménage. Enfin, il évalue les impacts à court et à plus long terme sur la sécurité alimentaire (production agricole), l'emploi, l'investissement et le bien être total des deux politiques tarifaires.

\section{La matrice de comptabilité sociale du Sénégal (MCS, 1996)}

Le calibrage du modèle d'équilibre général calculable nécessite la construction d'une matrice de comptabilité sociale (MCS) de l'économie sénégalaise. Celle-ci permet de décrire la structure de l'économie en illustrant l'ensemble des interdépendances entre les différents secteurs productifs et les agents (ménages, État et Reste du monde). Nous considérons que la ressource brute, le facteur eau primaire est détenue par l'État qui fournit le secteur du riz irrigué et de la production d'eau potable. Ce dernier combine cette ressource au travail et au capital pour produire une eau de qualité supérieure, l'eau potable. Cette dernière est alors distribuée par trois canaux (branchement privé, borne-fontaine et informel) pour être consommée soit en tant qu'intrant par les autres secteurs (agricoles, industriels et services) soit en tant que bien final par les ménages.

La MCS développée pour calibrer le modèle est une version à la fois agrégée et désagrégée des MCS construites par Dansokho et Diouf (1999) et Cabral (2005). Les données macroéconomiques proviennent du tableau entrée-sortie (TES), du tableau équilibre ressources-emplois par produit (TERE) de l'année 1996. Les informations sur les revenus et la consommation sont issues de l'Enquête sénégalaise auprès des ménages (Esam, 1995). Les données sur l'eau proviennent de la Sones, de la Sde et de la Direction de l'hydraulique (2003 et 2004). Enfin, la désagrégation du poste énergie en électricité-gaz, production d'eau potable, distribution formelle d'eau potable via le branchement privé, distribution formelle d'eau potable via la borne-fontaine et distribution informelle d'eau potable, émane de données issues de plusieurs enquêtes de terrain menées au Sénégal et à Dakar (Diagne et alii, 2004 ; Briand, 2006b).

La MCS comporte quinze secteurs (voir annexe 1). Elle se concentre sur les secteurs utilisateurs de la ressource en eau. Compte tenu du choix d'un critère d'intensité en eau ${ }^{(12)}$, elle distingue les secteurs 
agricoles intensifs en eau tels que le riz irrigué, le riz pluvial, le maraîchage, la pêche, des secteurs agricoles non intensifs en eau. Elle comporte un secteur de production d'eau potable à mission de service public (Sones). Plus encore, elle intègre trois secteurs de distribution d'eau potable (branchement privé, borne-fontaine et charretiers informels). Enfin, elle distingue les industries et services marchands intensifs en eau c'est-à-dire, fortement consommateurs de la ressource en tant qu' intrant de production, des industries et services marchands non intensifs en eau. Le dernier secteur est celui des services non marchands. On distingue quatre facteurs de production : le travail, le capital, la terre et l'eau primaire.

La MCS comporte huit catégories de ménages (voir annexe 2) selon leur zone géographique au Sénégal et, selon l'offre d'eau potable dont ils bénéficient: Dakar, les Autres centres urbains (ACU), le Bassin arachidier (BA), Niayes (NIAY), la Casamance (CASA), la Zone sylvo-pastorale (ZSP), le Sénégal oriental (SO), et le Fleuve hors delta (FLEUV). Six catégories de ménages ruraux sont distinguées selon les zones agroécologiques du Sénégal. Cet éclatement se justifie d'une part, par le fait que les zones rurales sont marquées par de fortes disparités en termes de potentialités agroclimatiques, d'infrastructures, de pratiques culturales, d'intensité dans l'utilisation des facteurs de production et en termes de sources de revenus des ménages (Cabral, 2005) et d'autre part, par la structure de consommation d'eau potable différente selon les régions. Les autres agents pris en compte sont les firmes, l'État et le Reste du monde.

\section{Le modèle}

Le modèle s'inspire du modèle statique néoclassique Exter développé par Decaluwé et alii (2001) mais diffère de celui-ci sur de nombreux aspects. Tout d'abord, le modèle comporte quatre facteurs de production (travail, capital, terre et eau primaire) contrairement à Exter qui en compte deux (travail et capital). La typologie des ménages diffère. Le modèle intègre une fonction linéaire des dépenses (LES) avec un panier minimal de consommation pour chaque catégorie de ménages, alors que dans Exter la consommation est une proportion fixe du revenu disponible. Les transferts inter-ménages et entre ces derniers et le Reste du monde sont explicitement pris en compte. Compte tenu des caractéristiques du secteur de production d'eau potable, nous modélisons un monopole naturel à rendements d'échelle croissants. Nous introduisons un salaire d'efficience et une fonction de chômage involontaire endogène. Enfin, il s'agit d'un modèle dynamique séquentiel récursif.
Le modèle comporte dix blocs d'équations : la production d'eau potable à rendements d'échelle croissants, la production des autres secteurs (en concurrence pure et parfaite), les revenus et l'épargne, les taxes, la demande, les échanges extérieurs, les prix, les conditions d'équilibre sur le marché du travail (avec chômage), les autres conditions d'équilibre et les équations dynamiques. Nous ne présentons que les blocs originaux du modèle (production d'eau potable, conditions d'équilibre sur le marché du travail et les équations dynamiques). Les équations des autres blocs plus standards ne sont pas présentées dans le cadre de cet $\operatorname{article}^{(13)}$

La production d'eau potable $\left(X S_{\text {WAT }}\right.$ ) est modélisée en deux étapes (équation (1) du bloc 1). Tout d'abord, le facteur travail $\left(L D_{\text {WAT }}\right)$ est combiné au facteur capital $\left(K D_{W A T}\right)$ par une fonction de Cobb-Douglas à rendements d'échelle constants pour donner un facteur composite capital-travail $\left(K L_{W A T}\right)$. Puis, ce facteur composite est combiné au facteur eau primaire $\left(E D_{W A T}\right)$ par une fonction de Cobb-Douglas à rendements d'échelle croissants $\left(s_{W A T}\right)$ pour donner la valeur ajoutée $\left(V A_{W A T}\right)$. Nous faisons l'hypothèse que la valeur ajoutée est égale à la production d'eau potable, ce qui signifie qu'il n'y a pas de consommations intermédiaires (négligeables) (équation (1)). Ainsi, nous en déduisons les demandes optimales de facteurs à partir de la résolution des programmes de maximisation du profit. L'équation (2) donne l'écriture de la demande optimale du facteur composite capital-travail $\left(K L_{\text {WAT }}\right)$ du secteur de production d'eau potable. Les équations (3) et (4) donnent respectivement les écritures des demandes optimales des facteurs eau primaire $\left(E D_{W A T}\right)$ et travail du secteur de production d'eau potable $\left(L D_{W A T}\right)$. L'équation (5) donne le prix du facteur composite capital-travail $\left(P_{K L}\right)$. Compte tenu que la production d'eau potable est égale à la valeur ajoutée du secteur, l'équation (6) indique que le prix de la valeur ajoutée $\left(P V_{W A T}\right)$ est égal au prix de production d'eau potable $\left(P_{W A T}\right)$ et l'équation (7) indique que le prix de la valeur ajoutée $\left(P V_{W A T}\right)$ est aussi égal au coût moyen de production d'eau potable $\left(C M_{W A T}\right)$. À partir de la fonction de coût total $\left(C T_{\text {WAT }}\right)$ donnée par l'équation (10), nous calculons la fonction de coût moyen $\left(C M_{W A T}\right)$ (équation (9)) et de coût marginal de production d'eau potable $\left(\mathrm{Cm}_{\text {WAT }}\right)$ (équation (8)). À l'année de base, comme le prix de la valeur ajoutée est égal au coût moyen de production, l'État n'a pas à intervenir pour subventionner le secteur de production d'eau potable qui ne produit pas à perte. L'équation (11) indique que la subvention $\left(S U B_{W A T}\right)$ (égale à la différence entre le coût moyen et le coût marginal sur le volume total des ventes) est nulle à l'année de base. 
Bloc 1 : production d'eau potable (monopole naturel à rendements d'échelle croissants)

(1) $X S_{\text {WAT }}=V A_{W A T}$

(2) $K L_{W A T}=\left(\frac{\alpha_{W A T}^{K L E}}{s_{W A T}}\right)\left(\frac{C M_{W A T}}{P_{K L}}\right) V A_{W A T}$

(3) $E D_{W A T}=\left(\frac{s_{W A T}-\alpha_{W A T}^{K L E}}{s_{W A T}}\right)\left(\frac{C M_{W A T}}{r e_{1}}\right) V A_{W A T}$

(4) $L D_{W A T}=\left(\alpha_{W A T}^{K L} P_{K L} K L_{W A T}\right) / w$

(5)

$P_{K L}=\left(\frac{1}{A_{W A T}^{K L 1}}\right)\left(\frac{r_{W A T}}{\beta_{W A T}^{K L}}\right)^{\beta_{W A T}^{K L}}\left(\frac{w}{\alpha_{W A T}^{K L}}\right)^{\alpha_{W A T}^{K L}}$

(6) $P V_{W A T}=P_{W A T}$

(7) $P V_{W A T}=C M_{W A T}$

(8)

$$
\begin{gathered}
C m_{W A T}=\left(\frac{1}{s_{W A T}}\right) V A_{W A T}^{\frac{1}{s_{W A T}}-1} A_{W A T}^{K L E} P_{K L}^{\frac{\alpha_{W A T}^{K L E}}{s_{W A T}}} \\
r e_{1}^{1-\frac{\alpha_{W A T}^{K L E}}{s_{W A T}}}\left(\frac{\alpha_{W A T}^{K L E}}{s_{W A T}-\alpha_{W A T}^{K L E}}\right)^{1-\frac{\alpha_{W W T}^{K L E}}{s_{W A T}}}\left(\frac{s_{W A T}}{\alpha_{W A T}^{K L E}}\right)
\end{gathered}
$$

(9) $C m_{W A T}=\left(\frac{1}{s_{W A T}}\right) V A_{W A T}^{\frac{1}{S_{W A T}}-1} A_{W A T}^{K L E} P_{K L}^{\frac{\alpha_{W A T}^{K L E}}{S_{W A T}}}$

$$
r e_{1}^{1-\frac{\alpha_{W A T}^{K L E}}{s_{W A T}}}\left(\frac{\alpha_{W A T}^{K L E}}{s_{W A T}-\alpha_{W A T}^{K L E}}\right)^{1-\frac{\alpha_{W A T}^{K L E}}{s_{W A T}}}\left(\frac{s_{W A T}}{\alpha_{W A T}^{K L E}}\right)
$$

(10) $C T_{W A T}=\left(\frac{V A_{W A T}}{\alpha_{W A T}^{K L E}}\right)^{\frac{1}{s_{W A T}}} P_{K L}^{\frac{\alpha_{W A T}^{K L E T}}{\Phi_{W A T}}}$

$$
r e^{1-\frac{\alpha_{W A T}^{K L E}}{s_{W A T}}}\left(\frac{\alpha_{W A T}^{K L E}}{s_{W A T}-\alpha_{W A T}^{K L E}}\right)^{1-\frac{\alpha_{W A T}^{K L E}}{s_{W A T}}}\left(\frac{s_{W A T}}{\alpha_{W A T}^{K L E}}\right)
$$

(11) $S U B_{W A T}=\left(C M_{W A T}-C m_{W A T}\right) V A_{W A T}$

La production des autres secteurs (bloc 2) est modélisée en deux étapes. D'abord la production du secteur est exprimée comme une fonction de type Leontief combinant des parts fixes de la valeur ajoutée et des consommations intermédiaires. La valeur ajoutée est spécifiée de façon différente selon le type de secteur. Dans le secteur du riz irrigué, la valeur ajoutée est une fonction de Cobb-Douglas des facteurs travail, capital, terre et eau primaire. Dans les autres secteurs agricoles non irrigués (sauf la pêche), la valeur ajoutée est représentée par une fonction de Cobb-Douglas des facteurs travail, capital et terre. La valeur ajoutée des autres secteurs sauf celui des services non marchands est représentée par une fonction de Cobb-Douglas des facteurs travail et capital. En effet, l'extrant du secteur non marchand étant, par hypothèse, produit à partir du facteur travail, la valeur ajoutée de ce secteur est égale à la demande de travail. La demande de travail de chaque branche est déterminée par la condition de premier ordre de maximisation du profit pour les secteurs marchands. Les mêmes conditions d'optimisation déterminent la demande du facteur terre des secteurs agricoles et de l'eau dans le secteur du riz irrigué. En revanche, la demande de travail pour le secteur non marchand est déterminée par le rapport entre la valeur ajoutée et le prix unitaire du facteur travail. La consommation intermédiaire de chaque secteur est une part fixe de la production de ce secteur. La demande intermédiaire d'un produit par une branche est exprimée sous la forme d'une fonction linéaire la reliant avec la consommation intermédiaire de cette branche.

Les revenus et épargnes des agents (ménages, firmes et État) sont définis dans le bloc 3. La réaction des ménages aux divers chocs est étudiée à travers celle d'un ménage représentatif. Les ménages sont classés en huit catégories. Chaque catégorie de ménage reçoit une part fixe des revenus salariaux, des revenus du capital, de la rente de la terre et de la rémunération de l'eau primaire. La rente de la terre est en totalité allouée aux ménages ruraux et la part de la rémunération de l'eau primaire versée aux ménages est allouée seulement aux ménages de la Casamance et du Fleuve. Les ménages reçoivent aussi une proportion fixe des transferts versés par l'État, le reste du monde et les autres ménages ainsi que les dividendes versés par les firmes. Cet ensemble représente le revenu des ménages. Puis, le revenu disponible des ménages est représenté par ce revenu brut défalqué des impôts directs versés à l'État et des transferts versés aux autres ménages. L'épargne des ménages est une fonction linéaire du revenu disponible brut. Le revenu des firmes est constitué d'une part de la rémunération du capital agricole et du capital non agricole. Leur épargne est donnée par l'écart entre leur revenu brut et d'une part, les dividendes versés aux ménages et au reste du monde et d'autre part, les impôts directs versés à l'État. Le revenu de l'État ou les recettes publiques sont constituées de la somme de la part de la rémunération de l'eau primaire reçue par l'État, des taxes indirectes sur les ventes domestiques, des taxes à l'importation, des impôts directs collectés auprès des ménages et des firmes et, des transferts reçus du reste du monde. L'épargne publique apparaît de façon résiduelle comme la différence entre les revenus de l'État, ses dépenses, les transferts qu'il verse aux ménages et au reste du monde et, la subvention versée au secteur de production d'eau potable (nulle à l'année de base).

Le bloc 4 présente l'ensemble des taxes. Pour les biens échangeables, les impôts indirects intérieurs frappent d'une part, les importations à leur prix intérieur c'est-à-dire droits de douane compris, et d'autre part, la part non exportée de la production 
locale évaluée au prix reçu par le producteur. Pour les biens non échangeables, les recettes d'imposition indirecte portent sur la vente des biens et services produits localement. Les taxes à l'importation ou recettes douanières nettes à l'importation des biens échangeables sont une fonction linéaire reliant le taux de taxation douanière (net) à l'importation, le prix mondial à l'importation, le taux de change extérieur nominal et les quantités importées. L'impôt direct sur le revenu du ménage est une part fixe de son revenu déterminée par le taux d'imposition directe du revenu des ménages. L'impôt direct sur le revenu des firmes est également une proportion fixe de leur revenu. Cette dernière dépend également du taux d'imposition directe du revenu des firmes.

Le bloc 5 présente le module de demande. La consommation finale de chaque produit par les ménages est valorisée au prix du bien composite. Elle est une fonction de type $\operatorname{LES}^{(13)}$. Cette fonction permet de distinguer la consommation incompressible exogène, de la consommation discrétionnaire qui dépend du revenu. La consommation discrétionnaire est déterminée de façon endogène par les variations des prix et du revenu disponible du ménage (Decaluwé et alii, 2001). Le montant des dépenses de consommation est obtenu en retranchant du revenu disponible, l'épargne. Les dépenses de l'État sont données par la production en volume du secteur non marchand valorisée par son prix. La valeur de l'investissement par origine est une proportion fixe de l'investissement total. La demande intermédiaire d'un produit représente, quant à elle, la somme des demandes intermédiaires de ce produit par l'ensemble des branches de production.

Le bloc 6 présente les échanges extérieurs. On pose l'hypothèse de petit pays pour tous les produits de l'économie. On suppose que le producteur du produit échangeable peut affecter sa production totale, en proportion variable, à des ventes à l'exportation et à des ventes locales. Cette transformation entre les exportations et les ventes locales étant à élasticité de transformation commerciale constante et finie (finie et négative). Le ratio du volume offert localement et du volume exporté décroît avec l'augmentation du prix relatif que reçoit le producteur pour ses ventes à l'étranger et inversement. On suppose que les consommateurs des produits échangeables se voient offrir un volume total du produit composite qu'ils achètent, en proportion variable, au reste du monde et sur le marché intérieur. Leur choix entre les deux sources d'approvisionnement est exprimé par une fonction de substitution commerciale à élasticité de substitution commerciale constante et finie. Pour les biens non échangeables, on considère que les ventes locales sont égales à la production. L'offre totale est égale aux ventes locales. Enfin, l'épargne étrangère est égale au déficit courant de la balance des paiements extérieurs. Il s'agit de l'écart entre les ressources du reste du monde (importations, transferts reçus par le reste du monde et revenus du capital reçu par le reste du monde) et ses dépenses (exportations et transferts versés par le reste du monde).

Le bloc 7 définit les prix du modèle. Le prix de la valeur ajoutée des secteurs est égal à la valeur de la production nette des coûts intermédiaires rapportée au volume de la valeur ajoutée. La demande intermédiaire est exprimée aux prix du marché des produits composites. Le prix composite est celui qui détermine l'équilibre de l'offre totale du produit, qu'elle soit d'origine locale ou étrangère, et la somme de ses utilisations intérieures. Pour passer du prix international en devise du produit importé à son prix intérieur de marché, on introduit le taux de change extérieur nominal, les taux (nets) d'imposition indirecte douanière et intérieure. Le prix reçu par le producteur à l'exportation du produit est égal au prix international en monnaie nationale. Pour les biens échangeables, le prix du marché du produit local vendu sur le marché intérieur est égal à la différence entre la valeur de l'offre totale du produit au prix composite et celle de l'importation de ce produit à son prix intérieur, par unité vendue localement. Pour les biens non échangeables, le prix du marché du produit vendu sur le marché intérieur est égal à la valeur de l'offre totale du produit au prix composite par unité vendue localement. On retire du prix du marché du produit vendu sur le marché intérieur les taxes indirectes pour obtenir le prix reçu par le producteur pour ses ventes sur le marché intérieur. Pour les biens échangeables, le prix de production sur l'ensemble de ses ventes, qu'elles soient sur le marché intérieur ou à l'exportation est une moyenne pondérée du prix pour ses ventes sur le marché intérieur et du prix à l'exportation, les pondérations étant les parts vendues localement et à l'exportation du volume du produit. On calcule un indice général des prix qui est le prix du PIB au coût des facteurs, c'est-à-dire la somme des prix de la valeur ajoutée pondérés par les parts de la valeur ajoutée des différentes branches d'activité dans le PIB total. Enfin, on calcule la rémunération du capital dans les secteurs de la production d'eau potable, du riz irrigué, les autres secteurs agricoles non irrigués et les autres secteurs.

À partir des travaux de Annabi (2003), nous introduisons un salaire d'efficience et du chômage involontaire dans le modèle EGC (Shapiro et Stiglitz, 1984). Le taux de salaire dans l'économie est une relation inverse du taux de chômage (un). L'équilibre sur le marché de l'emploi (bloc 8) est un équilibre avec chômage involontaire. La contrainte incitative dépend de la désutilité de l'effort (ee), de la probabilité d'être renvoyé $(b b)$, de la probabilité de détection du tire au flanc $(q q)$, et du taux d'escompte $(r r)$. Elle est insérée dans le modèle en calibrant la désutilité de l'effort (ee) qui prend une valeur fixe dans les simulations. L'équation (12) donne l'équilibre sur le marché du travail où l'offre totale 
Bloc 8 : conditions d'équilibre sur le marché du travail (chômage)

(12) $L S=\sum_{j} L D_{j}+u n L S$

Équilibre sur le marché du travail

(13) $w=e e+(e e / q q)(b b / u n+r r)$

Contrainte incitative

$(L S)$ est égale à la somme des demandes de travail des branches $J\left(L D_{j}\right)$ et du chômage involontaire (au taux un). L'équation (13) décrit la détermination du salaire à partir de la contrainte incitative.

Les autres conditions d'équilibre du modèle sont les suivantes. Sur le marché des biens, l'offre du produit composite est la somme des demandes intermédiaires, des consommations finales des ménages et des demandes d'investissements en biens. L'offre des services marchands non intensifs en eau est égale à la somme des consommations finales des ménages, des demandes intermédiaires et des demandes d'investissements. L'offre totale de terre est égale à la somme des demandes de terre par les différents secteurs agricoles. L'investissement total est égal à la somme de l'épargne des ménages, des firmes, du gouvernement et de l'extérieur. Il s'agit d'un bouclage néoclassique. Nous calculons la variation équivalente des ménages afin d'évaluer la modification du bien-être des différentes catégories de ménages suite au choc. Enfin, nous vérifions si la solution du modèle satisfait à la loi de Walras.

Pour comparer les effets de court et long terme des chocs climatiques associés aux tarifications au coût moyen versus coût marginal, nous proposons une approche dynamique. Ce choix est primordial puisqu' il est possible que les effets de court terme soient sous-estimés par rapport aux effets de long terme. Dans la lignée des travaux de Annabi et alii, (2004) et Annabi et alii, (2005), nous développons un modèle dynamique séquentiel récursif. Ce type de modèle n'est pas le résultat d'optimisations intertemporelles des agents économiques (les agents sont myopes). Le modèle dynamique séquentiel est formulé comme un modèle statique qui est résolu séquentiellement sur toutes les périodes. Toutes les équations présentées ci-dessus sont indicées en $t$. Puis on introduit le bloc d'équations dynamiques suivant (bloc 10). À chaque période, le stock de capital est actualisé à partir d'une équation d'accumulation du capital. L'équation d'accumulation du capital (14) décrit la loi du mouvement du stock de capital sectoriel. On suppose implicitement que les stocks sont mesurés au début de la période et que les flux sont mesurés à la fin de la période. La fonction de demande d'investissement (16) détermine comment le nouvel investissement va être distribué entre les différents secteurs. Elle définit le taux d'investissement par destination.
Cette fonction de demande d'investissement que nous utilisons est proche de celle proposée par Bourguignon et alii, (1989). Le taux d'accumulation du capital, rapport de l'investissement $I N D_{T R, T}$ sur le stock de capital $K D_{T R, T}$ est augmenté en respectant le rapport du taux de rendement du capital $r_{T R, T}$ sur le coût d'usage du capital $U_{T}$. En introduisant l'investissement par destination, on doit respecter la condition d'égalité avec l'investissement total par origine de la MCS. Le coût d'usage du capital (17) est égal au prix de renouvellement du capital (ou indice du prix de l'investissement) multiplié par la somme du taux de dépréciation du capital et du taux d'intérêt réel (exogènes). L'offre totale de travail (15) est une variable endogène bien qu'elle soit simplement supposée croître au taux de croissance exogène de la population. L'équation (18) représente le coût de renouvellement du capital ou l'indice du prix de l'investissement. Il est calculé en faisant la somme des produits des prix composites des différentes biens et de la part des biens dans l'investissement total. Enfin, les variables exogènes telles que la consommation publique, les consommations incompressibles des ménages et les transferts croissent au même rythme que la population.

\section{Bloc 10 : équations dynamiques}

(14) $K D_{T R, T+1}=(1-\delta) K D_{T R, T}+I N D_{T R, T}$

Accumulation du capital

(15) $L S_{T+1}=(1+n) L S_{T}$

Croissance de la main d'œuvre

(16) $\frac{I N D_{T R, T}}{K D_{T R, T}}=\gamma_{1 T R}\left(\frac{r_{T R, T}}{U_{T}}\right)^{2}+\gamma_{2 T R}\left(\frac{r_{T R, T}}{U_{T}}\right)$

Demande d'investissement

(17) $U_{T}=P k_{T}(i r+\delta)$

Coût d'usage du capital

(18) $P k_{T}=\sum_{T R}\left(P c_{T R, T} \mu_{T R}\right)$

Coût de renouvellement du capital

(19) $I T=p k_{T} \sum_{T R} I N D_{T R, T}$

Équilibre de l'investissement

Le modèle résolu sur 20 périodes comporte au total 13306 équations pour 13306 variables endogènes et 2255 variables exogènes (résolu sous Gams). Le bouclage du modèle est le suivant. Conformément à l'hypothèse de petit pays, les prix mondiaux des importations et des exportations sont aussi supposés donnés. On suppose la consommation publique fixe. L'offre totale de facteur terre est supposée exogène. Les demandes de facteur eau primaire par les secteurs du riz irrigué et de la production d'eau 
potable sont fixes. Enfin les dividendes et les transferts entre les différents agents sont exogènes. Compte tenu des accords relatifs à l'intégration monétaire des pays de l'Union Économique et Monétaire de l'Afrique de l'Ouest (UEMOA) à laquelle appartient le Sénégal, le taux de change est le numéraire du modèle. Il convient de préciser que l'épargne des agents domestiques (ménages, firmes et État) ainsi que l'épargne étrangère (balance commerciale) sont endogènes. La dernière hypothèse signifie que la politique intérieure peut, en partie, être financée par l'étranger.

Pour le calibrage, les élasticités-revenu ainsi que les paramètres de Frisch (fonction LES) sont tirés des travaux de Dorosh (1996) sur Madagascar. Les élasticités de substitution des équations commerce extérieur proviennent de la base de données du Gtap et sont relatives à la zone de l'Afrique subsaharienne. Les élasticités de transformation sont les mêmes que celles utilisées par Cabral (2005). Les prix mondiaux des importations et des exportations de même que le taux de change, sont fixés à 1 à l'année de référence. Il en est de même du taux de salaire, du prix du capital, de la terre et de l'eau primaire. On a fixé les économies d'échelle dans la fonction de production d'eau potable à 1,3 . Le taux de chômage à l' année de base est fixé à $10 \%$ et les autres paramètres de la contrainte incitative sont fixés respectivement à $10 \%, 30 \%$ et $5 \%$ (Annabi, 2003). Le taux de croissance de la population ( $n$ ) est fixé à $2 \%$, le taux de dépréciation du capital $(\delta)$ à $3 \%$ et le taux d'intérêt réel (ir) à 4\% (Annabi et alii, 2004). Toutes les autres valeurs portant sur les variables du modèle sont tirées de la matrice de comptabilité sociale du Sénégal de 1996. En statique, l'analyse est faite par rapport à l'année de base représentée par la MCS. En dynamique, l'économie crô̂t naturellement sans choc de politique. L'analyse est donc faite sur la base du sentier de croissance en l'absence de choc $^{(14)}$.

\section{Résultats des simulations des politiques tarifaires au coût moyen et marginal associées au scenario climatique}

Les deux simulations portent sur le même scénario climatique. Celui-ci permet de représenter l'évolution dynamique et de long terme de la disponibilité des ressources en eau du Sénégal. Nous simulons un accroissement de $30 \%$ de la quantité de facteur eau primaire utilisée par les secteurs du riz irrigué et de la production d'eau potable sur les trois premières périodes $(1,2$ et 3$)$; puis nous simulons une chute de $30 \%$ de la quantité de facteur eau primaire utilisée par les secteurs du riz irrigué et de la production d'eau potable sur les périodes 7,8 et 9 . Toutes les autres périodes sont caractérisées par une stabilité climatique en terme de disponibilité des ressources en eau. Ce qui distingue les deux simulations, est la politique tarifaire de gestion de la demande en eau qui accompagne ces chocs climatiques. Le premier scénario est celui d'une politique tarifaire au coût moyen. Le second est celui de la mise en place d'une tarification au coût marginal accompagnée d'une subvention versée par l'État au secteur de production d'eau potable. Nous comparons les effets de court terme (1996) et de long terme (2015) de ces deux politiques associées au choc climatique sur l'économie sénégalaise (voir tableau 2 pour les principaux résultats).

\section{La tarification au coût moyen face aux chocs climatiques}

\section{À court terme (1996)}

La hausse de la disponibilité de la ressource primaire à court terme profite directement au secteur de production d'eau potable et du riz irrigué. Tout d'abord, elle entraîne une baisse du coût moyen, du coût marginal et du prix de production et de

Tableau 2 : principaux résultats des deux simulations

\begin{tabular}{|c|c|c|c|c|}
\hline \multirow[b]{2}{*}{ \% de variation par rapport au sentier de croissance sans choc } & \multicolumn{2}{|c|}{1996} & \multicolumn{2}{|c|}{2015} \\
\hline & $\begin{array}{c}\text { Tarification au } \\
\text { coût moyen }\end{array}$ & $\begin{array}{l}\text { Tarification au } \\
\text { coût marginal }\end{array}$ & $\begin{array}{c}\text { Tarification au } \\
\text { coût moyen }\end{array}$ & $\begin{array}{l}\text { Tarification au } \\
\text { coût marginal }\end{array}$ \\
\hline Coût moyen de production d'eau potable & $-35,6$ & $-32,36$ & 17,88 & 19,59 \\
\hline Coût marginal de production d'eau potable & $-35,6$ & $-32,36$ & 17,88 & 19,59 \\
\hline Prix à la consommation d'eau au branchement privé & $-10,82$ & -14 & 7,36 & $-2,96$ \\
\hline Prix à la consommation d'eau à la borne fontaine & $-9,24$ & -12 & 7,4 & $-2,91$ \\
\hline Prix à la consommation d'eau informelle & 0,64 & 1,08 & $-0,47$ & 0,23 \\
\hline Rémunération de l'eau primaire dans le secteur du riz irrigué & $-23,75$ & $-23,47$ & 57,98 & 59,48 \\
\hline Rémunération de l'eau primaire dans le secteur de la production d'eau potable & $-49,04$ & $-45,88$ & 95,28 & 107,27 \\
\hline Taux de salaire & 0,67 & 1,13 & $-0,55$ & 0,29 \\
\hline Rémunération de la terre & 0,43 & 0,88 & $-0,89$ & 0,5 \\
\hline Epargne publique & $-1,89$ & $-5,82$ & $\mathbf{0 , 0 8}$ & $-9,74$ \\
\hline Investissement total & 1,03 & 1,84 & $-1,06$ & 0,79 \\
\hline Taux de chômage & $-0,9$ & $-1,5$ & $\mathbf{0 , 8 5}$ & $-0,43$ \\
\hline Bien être total & $\mathbf{0 , 8 1}$ & 1,11 & $-0,77$ & $-0,18$ \\
\hline
\end{tabular}

Source : calculs de l'auteur. 
consommation de $35,6 \%$. Cette chute du coût de l'intrant eau potable bénéficie directement aux secteurs de distribution d'eau potable. En effet les prix de production et de consommation de l'eau au branchement privé et à la borne-fontaine diminuent respectivement de $10,82 \%$ et 9,24\%. La hausse de la quantité d'eau primaire disponible génère alors une hausse de la production des secteurs du riz irrigué $(+8,92 \%)$, de la production d'eau potable $(+2,9 \%)$, de la distribution au branchement privé $(+1,78 \%)$ et à la borne-fontaine $(+9,38 \%)$.

Compte tenu de la fonction de production de Leontief de ces secteurs, leurs consommations intermédiaires respectives augmentent dans les mêmes proportions. Il y a donc un effet d'entraînement sur les autres secteurs dont la production augmente aussi mais dans de moindres proportions (sauf la pêche et l'industrie intensive en eau). On a donc globalement une expansion des secteurs qui répond aussi à une hausse de la demande via ses trois composantes : les demandes intermédiaires, la consommation finale des ménages et les demandes d'investissement. Cette hausse de la demande s'illustre par une hausse des importations (sauf pour le riz irrigué dont la production locale suffit pour répondre à la demande). Elle s'explique tout d'abord par la hausse des demandes intermédiaires liée à l'expansion des secteurs et la baisse du prix de l'eau potable mais aussi, par une hausse des revenus des agents. Le revenu des agents a en effet augmenté compte tenu de l'augmentation des rémunérations factorielles. En effet, le salaire et le rendement de la terre s' accroissent respectivement de $0,67 \%$ et $0,43 \%$, la rémunération du capital ayant augmenté aussi dans tous les secteurs (sauf dans celui du riz irrigué et de la production d'eau potable).

En effet, dans ces deux derniers secteurs, l'eau primaire s'est substituée aux autres facteurs (travail, capital et terre) qui ont donc pu être absorbés par les autres secteurs qui doivent assurer leur expansion. Notamment, les secteurs de distribution d'eau potable bénéficient largement de la libération de la main-d'œuvre, en particulier le secteur des bornes-fontaines intensif en travail. Les autres secteurs agricoles bénéficient aussi de la libération de la terre par le secteur du riz irrigué. C'est le maraîchage (secteur agricole le plus en expansion après le riz irrigué compte tenu de la baisse du coût de l'intrant eau) qui accapare la plus grande partie de la terre $(+1,1 \%,+0,86 \%$ et $+0,3 \%$ respectivement pour le maraîchage, le riz pluvial et l'autre agriculture).

La demande d'exportation de riz irrigué et de maraîchage croissent alors respectivement de 23,9\% et $0,37 \%$. L'expansion des secteurs intensifs en eau se traduit aussi par une hausse de leur investissement par destination : $+1,61 \%,+2,48 \%,+15,79 \%$ et $+42,22 \%$ respectivement pour le maraîchage, l'énergie, le branchement privé et la borne-fontaine.
Au niveau des ménages, le choc combiné au maintien de la politique actuelle au coût moyen génère à court terme une hausse du bien-être de tous les ménages sauf ceux du Fleuve (baisse de 0,07\%). La hausse du bien-être des ménages urbains est plus importante $(+0,51 \%$ pour Dakar et $+0,23 \%$ pour les autres centres urbains) que celle des ruraux $(+0,02 \%$ en moyenne).

$\mathrm{Au}$ niveau macroéconomique, bien que l'épargne gouvernementale baisse (du fait de la chute des rémunérations de l'eau primaire), l'investissement total s'accroît de 1,03\%. Ceci s'explique par la hausse des épargnes des autres agents (ménages et entreprises) liée à la hausse de leur revenu issu des autres facteurs dont la rémunération a augmenté. Ainsi, le bien être total s'accroît de $0,81 \%$ (compte tenu de la hausse des revenus et de la consommation) et le taux de chômage diminue de $0,9 \%$ grâce à l'expansion de l'économie sénégalaise. Enfin en terme de distribution d'eau potable, la politique d'accroissement de l'offre génère à court terme une substitution de la borne fontaine $(+9,38 \%)$ au branchement privé $(+1,78 \%)$ et du formel à l'informel $(+0,56 \%)$.

\section{À long terme (2015)}

Dix ans après le choc de déficit hydrique subit sur trois périodes, le coût moyen, le coût marginal et les prix de production et de consommation de l'eau potable ont augmenté de $17,88 \%$. Ceci se traduit par une hausse du coût de l'intrant eau potable par les autres secteurs utilisateurs.

Cette baisse de la disponibilité de la ressource a généré une chute de la production d'eau potable de $3,61 \%$ et du riz irrigué de $13,9 \%$. La baisse de la production d'eau potable combinée à la hausse du prix de l'eau potable génère une chute de la production des secteurs intensifs en eau $(-0,54 \%$; $-0,58 \% ;-0,77 \% ;-0,79 \% ;-0,99 \% ;-2,48 \%$ et $-10,1 \%$ respectivement pour l'industrie et les services marchands intensifs en eau, l'énergie, la distribution informelle d'eau, le maraîchage, le branchement privé et la borne-fontaine). La distribution d'eau potable est donc très affectée. Compte tenu de la chute dans les mêmes proportions de leurs consommations intermédiaires, les autres secteurs par effet d'entraînement se contractent aussi.

La chute de la production du riz irriguée est en partie compensée par la hausse des importations $(+6,63 \%)$ mais il n'en est pas de même pour les autres biens et services dont les importations reculent. Ceci traduit une contraction de la demande domestique qu'il nous faut alors expliquer. En effet, dans le secteur du riz irrigué, la baisse de la disponibilité de la ressource eau primaire est compensée par un accroissement de la demande des autres facteurs. Le travail, le capital et la terre se substituent à l'eau primaire. Leurs demandes respectives augmentent de 0,83\%,0,44\% 
et $0,61 \%$. La hausse de la demande de terre par le riz irrigué se fait au détriment des autres secteurs agricoles surtout du maraîchage puisque fortement intensif en eau (très affecté par la hausse du prix de l'eau), c'est le secteur agricole après le riz irrigué qui se contracte le plus. De même, dans le secteur de production d'eau potable, la baisse de la disponibilité de la ressource eau primaire est compensée par un accroissement de la demande des facteurs travail et capital $(+24,35 \%$ et $+23,38 \%)$ au détriment des autres secteurs. Cette baisse des demandes de facteurs par les autres secteurs est due à leur contraction.

Au niveau des ménages, on observe à long terme, compte tenu du déficit hydrique, une dégradation du bien-être des ménages sauf pour Niayes (reste constant) et pour le Fleuve qui augmente légèrement. La perte de bien-être des ménages urbains (-0,44\% pour Dakar et $-0,21 \%$ pour les autres centres urbains) est plus forte que celle des ruraux $(-0,03 \%$ en moyenne).

$\mathrm{Au}$ niveau macroéconomique à long terme, on observe une contraction générale des secteurs. Malgré une hausse des rémunérations de l'eau primaire qui entraîne une hausse de l'épargne gouvernementale, la chute des rémunérations des autres facteurs (salaire et rendement du capital) génère une baisse du revenu des autres agents et donc de leur épargne. Ainsi, l'investissement et le bien-être total se dégradent respectivement de $1,06 \%$ et $0,77 \%$ et le taux de chômage augmente de $0,85 \%$. En terme de distribution d'eau potable, le déficit hydrique affecte sur le long terme bien plus la distribution via les bornes fontaines $(-10,1 \%)$ que celle via le branchement privé $(-2,48 \%)$. La distribution informelle est la moins affectée $(-0,79 \%)$ puisqu' elle joue alors un rôle de régulation face à la pénurie.

\section{La tarification au coût marginal face aux chocs climatiques}

On suppose que l'État décide de mettre en place une politique de tarification de l'eau potable au coût marginal. On pose alors un prix de production de l'eau potable non plus égal à son coût moyen (1 à l'année de base) mais égal à son coût marginal $(0,769)$. Pour maintenir la survie du secteur, l'État lui verse une subvention pour compenser la perte.

\section{À court terme (1996) :}

La hausse de la disponibilité de la ressource permise par les politiques d'accroissement de l'offre cumulée à la mise en place d'une politique de tarification au coût marginal entraîne à court terme une chute des coûts moyen et marginal de production d'eau potable de $32,36 \%$. Les prix de production et de consommation d'eau potable diminuent plus que dans le premier scénario $(-47,97 \%$ contre $-35,6 \%)$. Il s'en suit une plus forte baisse du coût de l'intrant eau potable pour les secteurs utilisateurs. En effet, les prix de l'eau potable distribuée par le branchement privé et à la borne fontaine baissent plus que dans le premier scénario (respectivement $-14 \%$ contre $-10,82 \%$ et $-12 \%$ contre $-9,24 \%$ ).

Ainsi, la hausse de la production des secteurs de l'eau potable est aussi accentuée $(+4,01 \%$ contre $+2,9 \%$ pour la production d'eau potable, $+2,45 \%$ contre $+1,78 \%$ pour la distribution au branchement privé, $+13 \%$ contre $+9,38 \%$ pour la distribution à la borne fontaine). Ceci signifie que la hausse de leurs consommations intermédiaires évolue aussi dans des proportions plus fortes que dans le premier scénario avec un effet d'entraînement sur les autres secteurs plus fort aussi. C'est pourquoi les hausses des productions des autres secteurs se font aussi dans de plus fortes proportions. Cette hausse de l'offre répond à une demande de court terme elle-même en hausse. En effet, l'expansion des secteurs intensifs en eau, via la hausse des demandes intermédiaires et la baisse du prix de l'eau potable explique cette évolution à la hausse de la demande totale.

Cette dernière est assurée par l'augmentation des revenus des agents provoquée par la hausse des rémunérations factorielles plus conséquente que dans le premier scénario : le taux de salaire et le rendement de la terre croissent respectivement de $1,13 \%$ contre $0,67 \%$ et $0,88 \%$ contre $0,43 \%$. De même, le rendement du capital augmente plus dans tous les secteurs sauf dans le riz irrigué et la production d'eau potable où il diminue conformément au premier scénario. Ces évolutions des rémunérations factorielles s'expliquent par le fait que dans les secteurs du riz irrigué et de la production d'eau potable, l'eau primaire se substitue aux autres facteurs absorbés par les autres secteurs en expansion. Les hausses des demandes de travail dans le secteur du branchement privé et de la borne fontaine sont plus fortes (respectivement $+13,9 \%$ et $+36,53 \%$ contre $+9,95 \%$ et $+25,65 \%$ ). Dans les secteurs agricoles, la terre libérée par le riz irrigué $(-1,38 \%$ contre $-1,29 \%$ dans le premier scénario) est encore plus accaparée par le riz pluvial $(+1,21 \%$ contre $+0,86 \%)$ et le maraîchage $(+1,4 \%$ contre $+1,1 \%)$.

La hausse des investissements des secteurs par destination est aussi plus forte pour le riz pluvial, le maraîchage, l'autre agriculture, l'industrie non intensive en eau, l'énergie, le branchement privé, la borne fontaine et les services. Plus particulièrement, l'investissement dans le secteur du branchement privé s' accroît de $22,4 \%$ contre $15,79 \%$ et de $62,26 \%$ contre $42,22 \%$ dans celui de la borne fontaine. L'effet de la mise en place d'une tarification au coût marginal est à court terme plus bénéfique en termes d'investissements dans les secteurs de distribution d'eau potable. 
Au niveau des ménages, le choc combiné à la mise en place d'une politique tarifaire au coût marginal génère à court terme une amélioration du bien-être de tous les ménages sauf ceux du Fleuve $(-0,05 \%)$. Cette hausse du bien-être est comme dans le premier scénario plus forte pour les urbains $(+0,68 \%$ pour Dakar et $+0,31 \%$ pour les autres centres urbains) que pour les ruraux (+0,4\% en moyenne), mais dans de plus amples proportions. Ceci signifie que la mise en place d'une tarification au coût marginal accentue les effets bénéfiques du choc sur le bien-être des ménages.

Au niveau macroéconomique, la mise en place d'une tarification au coût marginal fait moins baisser les rémunérations de l'eau primaire. Le versement par l'État d'une subvention visant à combler le déficit budgétaire du secteur de production d'eau potable génère une dégradation de l'épargne gouvernementale de 5,82\% contre 1,89\% lorsque l'État n'intervient pas dans le premier scénario. Mais ceci n'entrave pas le niveau d'investissement total bien au contraire. Ce dernier augmente de 1,84\% contre $1,03 \%$. Ceci s'explique par la hausse des épargnes des autres agents qui fait plus que compenser la baisse de celle de l'État. Au final, l'expansion plus forte de l'économie avec la mise en place d'une tarification au coût marginal génère un plus fort accroissement du bien-être total $(+1,11 \%$ contre $+0,81 \%$ ) et une diminution plus importante du taux de chômage (-1,5\% contre $-0,9 \%)$. En terme de distribution d'eau potable, on observe les mêmes résultats que dans le premier scénario même si ceux-ci sont plus accentués. À savoir, une substitution de la borne fontaine $(+13 \%)$ au branchement privé $(+2,45 \%)$, ce qui signifie un fort développement du parc des bornes fontaines suite aux politiques d'accroissement de l'offre, et une substitution du formel à l'informel $(+0,74 \%)$.

\section{À long terme (2015)}

Nous observons que la mise en place d'une politique tarifaire au coût marginal permet à long terme de contrebalancer les effets négatifs sur l'économie du choc de déficit hydrique. En effet à long terme, les coûts moyen et marginal de production d'eau potable augmente de $19,59 \%$. Mais le prix de production et de consommation d'eau potable diminue de $7,82 \%$ tandis qu'il augmentait de 17,88\% dans le premier scénario. Les tendances de long terme s'inversent complètement selon que le gouvernement mène une politique de tarification au coût moyen ou au coût marginal. Ce résultat démontre qu'à long terme, une politique de tarification au coût marginal est encore bien plus bénéfique qu'à court terme. L'analyse de l'évolution des autres agrégats nous le démontre.

Malgré le choc de rareté de la ressource, le prix de l'eau potable produite diminue, ce qui se traduit par une baisse du coût de l'intrant eau potable pour les autres secteurs intensifs en eau. En effet, les prix de l'eau potable distribuée au branchement privé et à la borne fontaine diminuent respectivement de 2,96\% et $2,91 \%$ tandis que dans le premier scénario (tarification au coût moyen), ils augmentaient respectivement de $7,36 \%$ et $7,4 \%$.

Du coup, les effets sectoriels deviennent complètement différents. En effet, les productions des secteurs de la production d'eau potable, de la distribution au branchement privé, à la borne fontaine et du maraîchage augmentent respectivement de $1,36 \%, 0,8 \%, 4,63 \%$ et $0,11 \%$ tandis qu'elles diminuaient respectivement de $3,61 \%, 2,48 \%, 10,1 \%$ et $0,99 \%$ dans le premier scénario.

Ces résultats sont très intéressants car il s'en suit un effet d'entraînement positif sur la plupart des autres secteurs dont la production progresse aussi via les consommations intermédiaires. En effet, à part la production du riz irrigué qui chute pareillement au premier scénario $(-13,9 \%)$, seules les productions de pêche, d'autre agriculture et d'industrie intensive en eau diminuent ici mais, dans de moindres proportions. Ainsi l'expansion de la plupart des secteurs va de pair avec la hausse des rémunérations factorielles. Le taux de salaire et le rendement de la terre s'accroissent respectivement de $0,29 \%$ et $0,5 \%$ tandis qu'ils baissaient respectivement de $0,55 \%$ et $0,89 \%$ dans le premier scénario. Cette hausse des rémunérations factorielles justifie l'expansion des secteurs permise par la hausse de la demande totale via ses trois composantes (demande intermédiaire, consommation finale et demande d'investissement). En effet, le revenu et l'épargne des agents progressent sauf l'épargne gouvernementale qui se dégrade de 9,74\% (contre une légère hausse de $0,08 \%$ dans le cas de la tarification au coût moyen) du fait des subventions versées au secteur de production d'eau potable. Mais cette dégradation de l'épargne gouvernementale est plus que compensée par la hausse des épargnes des autres agents puisque au final, l'investissement total de long terme s'accroît de $0,79 \%$ alors qu'il diminuait de $1,06 \%$ dans le premier scénario.

Pour comprendre le financement de l'investissement total, le tableau 3 montre que l'épargne des firmes augmente de 2,4\% et l'épargne étrangère de 15,43\% avec la politique de tarification au coût marginal tandis qu'elles diminuaient respectivement de $3,01 \%$ et $4,91 \%$ avec la politique de tarification au coût moyen.

De même, le tableau 4 montre que l'épargne de tous les ménages progresse avec la politique de tarification au coût marginal tandis que seule l'épargne des ménages du Fleuve augmentait (compte tenu de la hausse de la rémunération de l'eau primaire) avec la politique de tarification au coût moyen. 
Tableau 3 : \% de variation de l'épargne des firmes (SE) et étrangère $(\mathrm{SR})$ par rapport à la tendance de long terme sans choc

\begin{tabular}{|l|c|c|}
\hline \multirow{2}{*}{$\%$ variation épargne } & \multicolumn{2}{|c|}{2015} \\
\cline { 2 - 3 } & $\begin{array}{c}\text { Tarification coût } \\
\text { moyen }\end{array}$ & $\begin{array}{c}\text { Tarification coût } \\
\text { marginal }\end{array}$ \\
\hline SE (firmes) & $-3,01$ & 2,4 \\
\hline SR (extérieure) & $-4,91$ & 15,43 \\
\hline
\end{tabular}

Source : calculs de l'auteur.

La hausse des différentes épargnes des agents explique alors l'évolution à la hausse de l'investissement total. Ce résultat est lié aux hypothèses de bouclage du modèle et plus particulièrement, à l'endogénéité de la balance commerciale qui rend possible le financement de la politique intérieure par le Reste du Monde. Cependant, le tableau 3 présenté en annexe 3 démontre que la structure de l'épargne totale et donc, du financement de l'économie reste quasi inchangée avec la mise en place de la tarification au coût marginal. Notamment, la part de financement de l'investissement total par l'épargne étrangère est de $10,11 \%$ dans le scénario de tarification au coût marginal contre $9,34 \%$ dans le scénario de tarification au coût moyen et $9,48 \%$ dans la tendance sans choc.

Le choc hydrique combiné à la mise en place d'une tarification au coût marginal provoque à long terme le maintien du bien-être des ménages de la zone sylvopastorale et une hausse de celui des ménages du Fleuve $(+0,04 \%)$, hausse plus importante que dans le premier scénario. Comme dans le scénario précédent, le choc génère une dégradation du bien-être de tous les autres ménages plus forte pour les urbains $(-0,1 \%$ pour Dakar et $-0,07 \%$ pour les autres centres urbains) que pour les ruraux $(-0,15 \%$ en moyenne), mais dans des proportions moindres. Ceci signifie que la mise en place d'une politique tarifaire au coût marginal permet d'atténuer l'effet négatif des pénuries d'eau sur le bien-être des ménages.

Enfin avec la mise en place d'une tarification au coût marginal, suite au choc de rareté de la ressource, le bien-être total se dégrade moins que dans le cas de la tarification au coût moyen $(-0,18 \%$ contre $-0,77 \%)$. L'expansion de la plupart des secteurs permise par la baisse du prix de l'eau relance l'activité économique et diminue le taux de chômage de $-0,43 \%$ tandis que ce dernier augmentait de $0,85 \%$ dans le premier scénario. En terme de distribution d'eau potable, on observe une tendance inverse au premier scénario puisque le déficit hydrique accompagné d'une politique tarifaire au coût marginal permet malgré tout à long terme, une expansion de la distribution avec une substitution de la borne fontaine $(+4,63 \%)$ au branchement privé $(+0,8 \%)$. Ceci s'explique par la dynamique d'investissement. En effet, les investissements dans les secteurs de l'eau augmentent tous contrairement au premier scénario. (respectivement $+33 \%,+2,06 \%$ et $+7,26 \%$ pour la production d'eau potable, la distribution au branchement privé et à la borne fontaine contre $+24,17 \%,-2,25 \%$ et $-10,7 \%$ ). Ainsi contrairement à la première simulation, le secteur informel ne joue plus le rôle de régulateur de la pénurie puisqu'il recule $(-0,06 \%)$.

\section{Conclusion}

Le modèle développé permet de simuler à court terme une phase d'accroissement de la disponibilité de la ressource (liée aux politiques d'accroissement de l'offre et au contexte pluviométrique actuel favorable) suivie d'une phase de déficits hydriques futurs conformément aux prévisions d'évolution de la demande et des risques d'apparition d'une nouvelle sécheresse au Sénégal. Ces chocs climatiques sont simulées de façon combinée à deux politiques tarifaires de gestion de la demande en eau : tarification au coût moyen versus coût marginal. Les résultats montrent que les effets très négatifs sur l'économie des pénuries d'eau pouvaient être contrecarrées avec la mise en place d'une politique tarifaire de gestion de la demande au coût marginal. Cette dernière permet de mieux gérer la rareté de la ressource. En effet le modèle montre que lorsque le Sénégal fait face aux déficits hydriques, la politique tarifaire au coût marginal inverse les tendances d'une économie qui maintiendrait une politique tarifaire au coût moyen. L'investissement total s'accroît, le bien être total se dégrade moins. La baisse du prix de l'eau (intrant et bien final), générée par le passage d'une tarification au coût moyen à une tarification au coût marginal, génère une expansion de nombreux secteurs, relance l'activité économique ce qui diminue le taux de chômage. La production agricole du riz pluvial et du maraîchage augmente tandis que tous les secteurs agricoles se contractent avec une tarification au coût moyen. Ce scénario tarifaire apparaît bénéfique à la lutte contre

Tableau 4 : \% de variation de l'épargne des ménages (SM) par rapport à la tendance de long terme sans choc

\begin{tabular}{|c|l|c|c|c|c|c|c|c|c|}
\hline \% de variation SM & Dakar & ACU & BA & NIAY & CASA & ZSP & \multicolumn{2}{c|}{ SO } & \multicolumn{2}{c|}{ FLEUV } \\
\hline \multirow{2}{*}{2015} & Tarification coût moyen & $-0,84$ & $-0,94$ & 0 & 0 & $-0,42$ & 0 & 0 & 0,78 \\
\cline { 2 - 21 } & Tarification coût marginal & 0,55 & 0,62 & 0 & 0 & 0,72 & 0 & 0 & 2,26 \\
\hline
\end{tabular}


l'insécurité alimentaire puisqu'en dépit des déficits hydriques, les productions agricoles du riz irrigué et du maraîchage s'accroissent. Enfin la distribution d'eau potable au branchement privé et aux bornes fontaines se développe grâce à la dynamique de l'investissement, ce qui accroît l'accès des ménages aux services améliorés. Le secteur informel de l'eau recule avec la mise en place d'une politique tarifaire au coût marginal tandis qu'il se développe en jouant pleinement son rôle de régulateur de la pénurie avec une politique tarifaire au coût moyen.

Ce résultat va à l'encontre des recommandations des bailleurs de fonds qui imposent au secteur de l'eau la contrainte d'équilibre budgétaire du secteur via une tarification au coût moyen. En effet, l'intervention de l'État via une subvention visant à combler le déficit du secteur lié à une tarification au coût marginal ne génère pas, dans le cadre d'une économie appliquée avec fiscalité distortante, d'effets négatifs. Au contraire, elle permet l'expansion de l'économie notamment grâce à la hausse des épargnes des agents domestiques et étrangers qui compense la dégradation de l'épargne gouvernementale.

\section{Notes}

(1) $100 \mathrm{FCFA}=0,15$ euros.

(2) Sonees : Société nationale des eaux du Sénégal.

(3) Sones : Société nationale des eaux du Sénégal.

(4) SDE : Société des eaux du Sénégal, filiale de la SAUR.

(5) Financés par une dizaine de bailleurs de fonds, dont la Banque mondiale (44\%).

(6) Financés à 52\% par la Banque mondiale.

(7) Les montants sont d'abord investis dans la station de traitement de Keur Momar Sarr (KMS) à Guéoul avec pour objectif de doubler la capacité de traitement de l'usine KMS en prévision de la demande à échéance 2007-2009.

(8) Management System Consultants Corp (1998) a produit l'étude de la demande en eau à l' horizon 2030 en collaboration avec les autorités sénégalaises, la Sones et la Direction de l'hydraulique.

(9) Taux de disponibilité : le temps de fonctionnement journalier est de 24 heures. Les coupures d'électricité, les opérations de maintenance, l'insuffisance des capacités de régulation de la production sont des facteurs de réduction du taux de disponibilité.

(10) L'économie et l'agriculture sénégalaises ont fait l'objet de quelques analyses en équilibre général calculable. Parmi ces travaux, on peut citer ceux de Dumont et Mesplé-Somps (2001), de Dansokho (2000), de Diagne et alii, (2002), de Boccanfuso et alii, (2003a), de Boccanfuso et alii, (2003b), de Cabral (2005) et enfin, de Annabi et alii, (2005). Ces modèles abordent la question des impacts des infrastructures (publiques et privées) sur la croissance et la compétitivité ; des impacts du Plan d'ajustement structurel et de la libéralisation commerciale du secteur agricole sur les inégalités et la pauvreté. Seuls les travaux de Dumont et Mesplé-Somps (2001) et de Annabi et alii, (2005) portent sur des modèles EGC dynamiques séquentiels. C'est cette approche dynamique que nous retenons pour notre modèle EGC appliqué à l'eau.

(11) Nous avons calculé, pour chaque branche, un coefficient d'intensité en eau égal au rapport entre la consommation intermédiaire d'eau utilisée par la branche et la somme des consommations intermédiaires totales de la branche. Puis, nous avons calculé pour les branches agricoles, industrielles et les services marchands un coefficient moyen d'intensité en eau (moyenne de tous les coefficients d'intensité en eau). Ces coefficients moyens d'intensité en eau sont devenus nos critères d'intensité en eau. Lorsque le coefficient d'intensité en eau d'une branche était inférieur au coefficient moyen, alors la branche était considérée comme non intensive en eau. Lorsque le coefficient d'intensité d'une branche était supérieur au coefficient moyen, alors la branche était considérée comme intensive en eau.

(12) Pour le détail des équations des autres blocs du modèle, voir : Briand (2006a).

(13) Connue aussi sous le nom de système linéaire des dépenses de type Stone Geary (Stone, 1954).

(14) Les graphiques représentant les résultats de la tendance sans choc et des simulations sont disponibles dans Briand (2006a). 


\section{Bibliographie}

Annabi N., Cissé F., Cockburn J. et Decaluwé B. (2005). "Trade liberalisation, Growth and Poverty in Senegal: A dynamic Microsimulation CGE Model Analysis", Working Paper, Cirpée.

Annabi N., Cockburn J. et Decaluwé B. (2004). "A Sequential Dynamic CGE Model for Poverty Analysis", mimeo, Cirpée-Pep.

Annabi N. (2003). "Modeling Labor Markets in CGE Models: Endogenous Labor Supply, Unions and Efficiency Wages", mimeo, Cirpée-Pep.

Berck A., Robinson S. et Goldman G. (1991). "The Use of Computable General Equilibrium Models to Assess Water Policies", in The Economics and Management of Water and Drainage in Agriculture, Kluwer Academic Publishers.

Boccanfuso D., Cabral Cissé F., Diagne A. et Savard L. (2003). «Pauvreté et distribution des revenus au Sénégal : une approche par la modélisation en équilibre général micro-simulé », Working Paper, n03-33, Cirpée-Pep.

Bourguignon F., Branson W.H. et De Melo J. (1989). "Macroeconomic Adjustment and Income Distribution: A Macro-Micro Simulation Model”, Technical Paper, $\mathrm{n}^{\circ} 1$, OECD.

Briand A. (2006a). " Marginal Cost versus Average Cost Pricing with Climatic Shocks in Senegal: a Dynamic Computable General Equilibrium Model Applied to Water », FEEM Working Paper $n^{\circ} 144.06$, Social Science Research Network.

Briand A. (2006b). "Politiques tarifaires de l'eau, sécurité alimentaire et vulnérabilité climatique au Sénégal : un modèle d'équilibre général calculable éclairé par une enquête ménages", Thèse de doctorat, Université de Rouen (France).

Brocklehurst C. et Janssens J.G. (2004). "Innovative Contracts, Sound Relationships: Urban Water Sector in Senegal", WSSB Discussion Paper Series, $\mathrm{n}^{\circ} 1$.

Burniaux J.M. et Truong T.P. (2002). “GTAP-E: An Energy Environmental Version of the GTAP Model", GTAP Technical Paper, $\mathrm{n}^{\circ} 16$.

Cabral F.-J. (2005). « Accord agricole et redistribution des revenus en milieu rural au Sénégal : essai de simulation à l'aide d'un modèle d'équilibre général calculable ", Thèse de doctorat en Sciences Économiques, Université Cheikh Anta Diop, Dakar.

Dansokho M. (2000). « Éssai de simulation de l'ajustement structurel dans le secteur agricole du Sénégal à l'aide d'un modèle d'équilibre général calculable », Thèse de doctorat d'État ès Sciences Économiques, Université Cheikh Anta Diop, Dakar.

Dansokho M. et Diouf A. (1999). « Élaboration des Matrices de Comptabilité Sociales pour les années 1992 et 1996 », Ministère de l'Économie et des finances, Unité de Politique Economique, République du Sénégal.

Decaluwé B., Martens A. et Savard L. (2001). Les politiques économiques du développement et les modèles d'équilibre général calculable, Les Presses de l'Université de Montréal.

Decaluwé B. et Patry A. (2001). «Quand le puits est à sec, on sait ce que vaut l'eau : le cas du Maroc », in Les politiques économiques du développement et les modèles d'équilibre général calculable, Les Presses de l'Université de Montréal, pp. 323-345.
Decaluwé B., Patry A. et Savard L. (1999). "When Water is no Longer Heaven Sent: Comparative Pricing Analysis in an AGE Model", Document de travail, n 9908, Créfa.

Decaluwé B., Patry A. et Savard L. (1998). «Quand l'eau n'est plus un don du ciel : un MEGC appliqué au Maroc », Revue d'Economie du Développement, n³-4, pp. 149-187.

Diagne A., Briand A. et Cabral F-J. (2004). “Sector Reforms and Universal Access to Water in Senegal", UNRISD Project on "Commercialization, Privatization and Universal Access to Water", Report, june.

Diagne A., Cabral J-F., Cissé F., Dansokho M. et Ba S. (2003). «Politiques commerciales, intégration régionale et distribution des revenus au Sénégal », Rapport MIMAP, Université Cheick Anta Diop, Dakar.

Dorosh P. (1996). " Structural Adjustment, Growth and Poverty in Madagascar: a CGE Analysis », monograph $\mathrm{n}^{\circ} 17$, NY: Ithaca Cornell University Press.

Dumont J.C.et Mesplé-Somps (2001). «Des retombées généralement bien appréciées : infrastructure publique, croissance et compétitivité au Sénégal ", in Les politiques économiques du développement et les modèles d'équilibre général calculable, Les Presses de l'Université de Montréal, pp. 399-418.

Enquête Sénégalaise Auprès des Ménages (Esam) (1995). République du Sénégal, Direction de la prévision et de la statistique, Dakar.

Horridge J-M., Dixon P-B. et Rimmer M-T. (1993). "Water Pricing and Investment in Melbourne: General Equilibrium Analysis with Uncertain Streamflow", Working Paper, ${ }^{\circ}$ IP-63, Center of Policy Studies and the Impact Project.

Gomez C.M., Tirado D. et Rey-Maquiera J.R (2004). "Water Exchanges versus Water Works: Insights from a Computable General Equilibrium Model for the Balearic Islands », Water Resources Research, n ${ }^{\circ}$, W10502 10. 1029/2004WR003235.

Goodman D-J. (2000). "More Reservoirs or Transfers ?: A Computable General Equilibrium Analysis of Projected Water Shortages in the Arkansas River Basin", Journal of Agricultural and Resource Economics, $\mathrm{n}^{\circ}$ 25(2), pp. 698-713.

Lofgren H. et Robinson S. (1997). "The MixedComplementarity Approach to specifying Agricultural Supply in Computable General Equilibrium Models", Washington D.C, IFPRI, TMD discussion papers.

Management System Consultants Corp (1998). « Rapport Stratégique - Annexe A - Étude de la demande », Approvisionnement en Eau Potable à Long Terme pour la région de Dakar, IFC.

Ndaw M.F. (2005). «Étude de cas : réforme du secteur de l'hydraulique au Sénégal : pièce maîtresse vers la réalisation des Objectifs du Millénaire pour le Développement », Ministère de l'Hydraulique, République du Sénégal, Dakar.

Robinson S. et Gehlar C. (1995). "Land, Water, Agriculture in Egypt: the Economywide Impact of Policy Reform", TMD discussion, IFPRI.

Sde (2004). "Evolution institutionnelle de l'hydraulique urbaine et de l'assainissement", Rapport diagnostic technique, République du Sénégal, Dakar, mars.

Seung C-K., Harris T-R., Englin, J-E. et Netusil N.R (2000). "Impacts of Water Reallocation: A Combined Computable General Equilibrium and Recreation Demand Model Approach”, The Annals of Regional Science, n³4, pp. 473-487. 
Shapiro C. et Stiglitz J-E. (1984). "Equilibrium Unemployment as a Worker Discipline Device", American Economic Review, ${ }^{\circ} 74$, pp. 433-444.

Sones (2004). « Rapport d'avancement du projet sectoriel eau au 31 décembre 2003 », Direction de la planification et de l'équipement, République du Sénégal, Dakar, janvier.

Sones (2002). « Études tarifaires - Modèle Castalia : rapport d'actualisation ", Direction du contrôle de l'exploitation, service contrôle administratif et commercial, République du Sénégal, Dakar, janvier.

Stone R. (1954). "Linear Expenditure Systems and Demand Analysis: An Application to the Pattern of British Demand", Economic Journal, n64, pp. 521-527.

Thabet C. (2003). « Réforme de la politique des prix de l'eau d'irrigation en Tunisie : approche en équilibre général », Thèse de Doctorat en Sciences économiques, ENSAR, Rennes. 
Tableau 1 : principales caractéristiques des secteurs (\%)

\begin{tabular}{|c|c|c|c|c|c|c|}
\hline \multirow[b]{2}{*}{ Secteurs } & \multirow{2}{*}{$\begin{array}{c}\text { Taux de valeur } \\
\text { ajoutée }\end{array}$} & \multicolumn{4}{|c|}{ Part des facteurs dans la valeur ajoutée } & \multirow{2}{*}{$\begin{array}{c}\text { Intensité en eau potable (part de } \\
\text { l'intrant eau dans la consommation } \\
\text { intermédiaire totale) }\end{array}$} \\
\hline & & Travail & Capital & Terre & $\begin{array}{c}\text { Eau } \\
\text { primaire }\end{array}$ & \\
\hline Riz irrigué & 91,14 & 12,28 & 44,48 & 9,46 & 33,78 & 0 \\
\hline Riz pluvial & 44,31 & 57,34 & 30,54 & 12,13 & 0 & 0 \\
\hline Maraîchage & 85,43 & 59,29 & 35,83 & 4,88 & 0 & 58,8 \\
\hline Pêche & 79,34 & 53,72 & 46,28 & 0 & 0 & 0,86 \\
\hline Autre agriculture & 77,02 & 61,43 & 32,75 & 5,81 & 0 & 0 \\
\hline Industrie intensive en eau & 42,53 & 18,63 & 81,37 & 0 & 0 & 5,3 \\
\hline Industrie non intensive en eau & 24,23 & 32,91 & 67,09 & 0 & 0 & 1,4 \\
\hline Autre énergie & 44,4 & 17,91 & 82,09 & 0 & 0 & 4,6 \\
\hline Production d'eau potable & 100 & 14,82 & 53,16 & 0 & 32,02 & 0 \\
\hline Distribution d'eau au branchement privé & 45,97 & 18,55 & 81,45 & 0 & 0 & 77 \\
\hline Distribution d'eau à la borne fontaine & 38,74 & 39,25 & 60,75 & 0 & 0 & 70,4 \\
\hline Distribution informelle d'eau & 98,95 & 88,65 & 11,35 & 0 & 0 & 100 \\
\hline Services marchands intensifs en eau & 61,05 & 44,2 & 55,8 & 0 & 0 & 2,6 \\
\hline Services marchands non intensifs en eau & 59,3 & 22,88 & 77,12 & 0 & 0 & 1,05 \\
\hline Services non marchands & 54,21 & 100 & 0 & 0 & 0 & 1,43 \\
\hline Total & 53,46 & 40,62 & 56,69 & 1,23 & 1,45 & 4,1 \\
\hline
\end{tabular}

Source : calculs de l'auteur, MCS, 1996.

Tableau 2 : répartition des sources de revenus des ménages selon les différents facteurs (\%)

\begin{tabular}{|c|c|c|c|c|c|}
\hline & Travail & Capital & Terre & Eau primaire & Total \\
\hline Dakar & 52,59 & 47,41 & 0 & 0 & 100 \\
ACU & 47,06 & 52,94 & 0 & 0 & 100 \\
BA & 21,49 & 68,09 & 10,42 & 0 & 100 \\
NIAY & 57,01 & 38,8 & 4,19 & 0 & 100 \\
CASA & 32,44 & 52,25 & 10,38 & 4,93 & 100 \\
ZSP & 30,25 & 62,68 & 7,07 & 0 & 100 \\
SO & 76,78 & 19,08 & 4,14 & 0 & 100 \\
FLEUV & 20 & 45,4 & 4,82 & 29,78 & 100 \\
Sénégal & 47 & 50,31 & 1,43 & 1,26 & 100 \\
\hline
\end{tabular}

Source : calculs de l'auteur, MCS 1996.

Tableau 3 : structure de l'épargne totale (\%) en 2015 de l'économie sans choc et de l'économie soumise aux déficits hydriques combinés aux deux politiques tarifaires (côut moyen versus coût marginal)

\begin{tabular}{|c|c|c|c|c|c|c|c|c|c|c|c|c|}
\hline Épargnes & Dakar & $\mathbf{A C U}$ & BA & NIAY & CASA & ZSP & SO & FLEUV & Firmes & État & RDM & Total \\
\hline Sans choc (en valeur) & 85963 & 35658 & 0 & 0 & 392 & 0 & 0 & 3360 & 21527 & 27207 & 18243 & 192352 \\
\hline Sans choc $($ en \%) & 44,69 & 18,54 & $\mathbf{0}$ & $\mathbf{0}$ & $\mathbf{0 , 2 0}$ & $\mathbf{0}$ & $\mathbf{0}$ & 1,75 & 11,19 & 14,14 & 9,48 & 100 \\
\hline Tarification coût moyen (en valeur) & 85443 & 35425 & 0 & 0 & 390 & 0 & 0 & 3380 & 21272 & 27215 & 17831 & 190957 \\
\hline Tarification coût moyen (en \%) & 44,74 & 18,55 & $\mathbf{0}$ & $\mathbf{0}$ & 0,2 & $\mathbf{0}$ & $\mathbf{0}$ & 1,77 & 11,4 & 14,25 & 9,34 & 100 \\
\hline Tarification coût marginal (en valeur) & 86301 & 35813 & 0 & 0 & 394 & 0 & 0 & 3418 & 21730 & 26177 & 19552 & 193385 \\
\hline Tarification coût marginal (en \%) & 44,63 & 18,52 & $\mathbf{0}$ & $\mathbf{0}$ & 0,2 & $\mathbf{0}$ & $\mathbf{0}$ & 1,77 & 11,24 & 13,54 & 10,11 & 100 \\
\hline
\end{tabular}

Source : calculs de l'auteur, MCS, 1996. 